NASA/CR-2002-211763

ICASE Report No. 2002-29

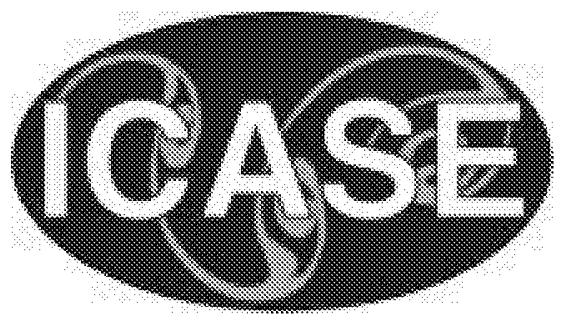

\title{
A Multi-domain Spectral Method for Supersonic Reactive Flows
}

Wai-Sun Don, David Gottlieb, and Jae-Hun Jung

Brown University, Providence, Rhode Island 


\section{The NASA STI Program Office ... in Profile}

Since its founding, NASA has been dedicated to the advancement of aeronautics and space science. The NASA Scientific and Technical Information (STI) Program Office plays a key part in helping NASA maintain this important role.

The NASA STI Program Office is operated by Langley Research Center, the lead center for NASA's scientific and technical information. The NASA STI Program Office provides access to the NASA STI Database, the largest collection of aeronautical and space science STI in the world. The Program Office is also NASA's institutional mechanism for disseminating the results of its research and development activities. These results are published by NASA in the NASA STI Report Series, which includes the following report types:

- TECHNICAL PUBLICATION. Reports of completed research or a major significant phase of research that present the results of NASA programs and include extensive data or theoretical analysis. Includes compilations of significant scientific and technical data and information deemed to be of continuing reference value. NASA's counterpart of peer-reviewed formal professional papers, but having less stringent limitations on manuscript length and extent of graphic presentations.

- TECHNICAL MEMORANDUM. Scientific and technical findings that are preliminary or of specialized interest, e.g., quick release reports, working papers, and bibliographies that contain minimal annotation. Does not contain extensive analysis.

- CONTRACTOR REPORT. Scientific and technical findings by NASA-sponsored contractors and grantees.
- CONFERENCE PUBLICATIONS. Collected papers from scientific and technical conferences, symposia, seminars, or other meetings sponsored or cosponsored by NASA.

- SPECIAL PUBliCATION. Scientific, technical, or historical information from NASA programs, projects, and missions, often concerned with subjects having substantial public interest.

- TECHNICAL TRANSLATION. Englishlanguage translations of foreign scientific and technical material pertinent to NASA's mission.

Specialized services that complement the STI Program Office's diverse offerings include creating custom thesauri, building customized data bases, organizing and publishing research results ... even providing videos.

For more information about the NASA STI Program Office, see the following:

- Access the NASA STI Program Home Page at http://www.sti.nasa.gov

- Email your question via the Internet to help@sti.nasa.gov

- Fax your question to the NASA STI Help Desk at (301) 621-0134

- Telephone the NASA STI Help Desk at (301) 621-0390

- Write to: NASA STI Help Desk NASA Center for AeroSpace Information 7121 Standard Drive Hanover, MD 21076-1320 
NASA/CR-2002-211763

ICASE Report No. 2002-29

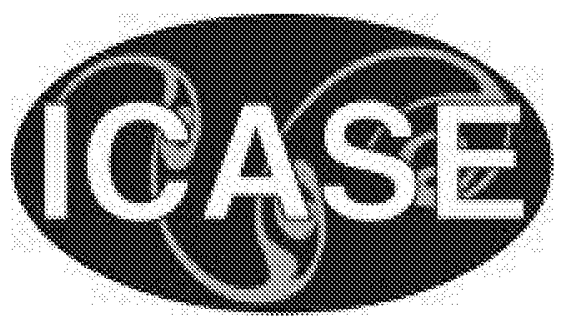

\section{A Multi-domain Spectral Method for Supersonic Reactive Flows}

Wai-Sun Don, David Gottlieb, and Jae-Hun Jung

Brown University, Providence, Rhode Island

ICASE

NASA Langley Research Center

Hampton, Virginia

Operated by Universities Space Research Association 
Available from the following:

NASA Center for AeroSpace Information (CASI)

7121 Standard Drive

Hanover, MD 21076-1320

National Technical Information Service (NTIS)

5285 Port Royal Road

Springfield, VA 22161-2171

(301) 621-0390

(703) $487-4650$ 


\title{
A MULTI-DOMAIN SPECTRAL METHOD FOR SUPERSONIC REACTIVE FLOWS *
}

\author{
WAI-SUN DON, DAVID GOTTLIEP \& JAE-HUN JUNG
}

\begin{abstract}
This paper has a dual purpose: it presents a multidomain Chebyshev method for the solution of the two-dimensional reactive compressible Navier-Stokes equations, and it reports the results of the application of this code to the numerical simulations of high Mach number reactive flows in recessed cavity. The computational method utilizes newly derived interface boundary conditions as well as an adaptive filtering technique to stabilize the computations. The results of the simulations are relevant to recessed cavity flameholders.
\end{abstract}

Key words. multi-domain spectral method, penalty interface conditions, supersonic combustor, recessed cavity flame-holder, compressible Navier-Stokes equations

Subject classification. Applied and Numerical Mathematics

1. Introduction. The efficacy of spectral methods for the numerical solution of highly supersonic, reactive flows had been previously reported in the literature. Don and Gottlieb $[7,8]$ simulated interactions of shock waves with hydrogen jets and obtained results showing the rich dynamics of the mixing process as well as the very complex shock structures. Don and Quillen [9] studied the interaction of a planar shock with a cylindrical volume of a light gas and showed that the spectral methods used gave good results for the flows with the shocks and complicated non-linear behaviors. In fact the results compared favorably to ENO schemes.

The methods reported above were based on Chebyshev techniques in one domain. In order to extend the utility of spectral methods to complex domains, multidomain techniques have to be considered. The main issue here is the stable imposition of the interface boundary conditions, and in this paper we consider mainly the penalty method, introduced for hyperbolic equations by Funaro and Gottlieb [10, 11].

There is an extensive literature on the subject: Hesthaven $[13,14,15]$ applied penalty $\mathrm{BC}$ for Chebyshev multidomain methods using the characteristic variables. Carpenter et. al. $[4,17,18]$ used it in conjunction with compact finite difference schemes, going from a scalar model equation to the full $\mathrm{N}$-S equations in general coordinate systems. Carpenter, Gottlieb and Shu [5] demonstrated the conservation properties of the Legendre multidomain techniques.

In the current work we follow the same methodology but in the context of supersonic combustion. We formulate the stable interface conditions based on the penalty method in a conservative form for both Euler and Navier-Stokes equations in two dimensional Cartesian coordinates. We derive stability conditions, independent on the local flow properties, for the penalty parameters for the Legendre spectral method. We also present here a new adaptive filtering technique that stabilize the spectral scheme when applied to supersonic reactive flows.

Implementing this method, we consider supersonic combustion problems in recessed cavities in order to establish the efficacy of recessed cavity flame-holders.

* Brown University, Division of Applied Mathematics, 182 George Street, Providence, RI 02912 (E-mail: wsdon, dig, jungorfm.brown.edu) This work was performed under AFOSR grant no. F49620-02-1-0113 and DOE grant no. DE-FG0296ER25346. This research was supported by the National Aeronautics and Space Administration while the second author was in residence at ICASE, NASA Langley Research Center, Hampton, VA 23681. 
We consider two different cases; (1) Non-reactive flows with two chemical species and (2)Reactive flows whth four chemical species.

Recessed cavities provide a high temperature, low speed recirculating region that can support the production of radicals created during chemical reactions. This stable and efficient flame-holding performance by the cavity is achieved by generating a recirculation region inside the cavity where a hot pool of radicals forms resulting in reducing the induction time and thus obtaining the auto-ignition [2, 22]. Fxperiments have shown that such efficiency depends on the geometry of the cavity such as the degree of the slantness of the aft wall and the length to depth ratio of cavity $L / D$. Thus one can optimize the flame-holding performance by properly adjusting the geometrical parameters of the cavity flame-holder system for a given supersonic flight regime. There are two major issues of such cavity fame-holder system that need to be investigated ; (1) What is the optimal angle of the aft wall for a given $L / D$ ? and (2) How does the fuel injection interact whth cavity flows? An answer to these questions require both a comprehensive laboratory and numerical experiments.

There have been previous numerical studies on these questions, many of them rely on the turbulence models. Rizzetta [19] used a modification of the Baldwin Lomax algebraic turbulence model. Davis and Bowersox [6] also used Baldwin-Lomax model. Zhang et.al. [23] used Wilcox $\kappa-\omega$ turbulence model. Baurle and Gruber [3] used the Menter model. Although the use of the turbulence models can make it possible to handle the compressible supersonic shear flows, the results are quite model-dependent as they require parametric assumptions. In this work, we solve the full compressible Navier-Stokes equations with chemical reactions without any turbulence model, using a multi-domain spectral method.

Results of several numerical studies including the present study have shown that the stability of the recirculation inside cavity is enhanced for the lower angle of cavity compared to the rectangular cavity. The present study, however, gives more accurate and finer details of the fields than those done by lower order numerical experiments. We show that a stationary recirculation region is not formed inside the cavity contrary to what the lower order schemes predict. A quantitative analysis made in this study shows that the lower angled wall of the cavity reduces the pressure fuctuations significantly inside the cavity for the non-reactive flows. We obtained a similar result for the reactive flows with the ignition of the fuel supplied initially in the cavity.

The rest of this paper is organized as follows. In section 2 the governing equations are given. In section 3 we describe the numerical method used in this work. In this section we present the adaptive-filtering used to remove the high frequency mode that causes the instability due to the non-smoothness of the flow, and we derive stable penalty interface conditions. In section 4 the system of the supersonic recessed cavity combustor is described. In section 5 the main results of this work are given and discussed.

2. The Governing Equations. In this work, we consider the compressible Navier-Stokes equations in the presence of the chemical reactions. Since Hydrogen is used as a fuel in our numerical experiments, four chemical species are considered, i.e. $\mathrm{H}_{2}, \mathrm{O}_{2}, \mathrm{H}_{2} \mathrm{O}$ and $\mathrm{N}_{2}$ with the chemical reaction between $\mathrm{Hydrogen}$ and Oxygen gases:

$$
2 \mathrm{H}_{2}+\mathrm{O}_{2} \rightleftharpoons 2 \mathrm{H}_{2} \mathrm{O}
$$

The two-dimensional compressible Navier-Stokes equations in conservative form can be written as:

$$
\frac{\partial q}{\partial t}+\frac{\partial F}{\partial x}+\frac{\partial G}{\partial y}=\frac{\partial F_{\nu}}{\partial x}+\frac{\partial G_{\nu}}{\partial y}+C
$$


The state vector, $q$ and the inviscid fluxes, $F$ and $G$ are given by

$$
q=\left(\begin{array}{c}
\rho \\
\rho u \\
\rho v \\
E \\
\rho \mathbf{f}
\end{array}\right), F=\left(\begin{array}{c}
\rho u \\
\rho u^{2}+P \\
\rho u v \\
(E+P) u \\
\rho \mathbf{f}_{\mathbf{u}}
\end{array}\right), G=\left(\begin{array}{c}
\rho v \\
\rho u v \\
\rho v^{2}+P \\
(E+P) v \\
\rho \mathbf{r}_{\mathrm{v}}
\end{array}\right)
$$

Here $\rho$ is the density, $u$ and $v$ are the mean mixture velocity components of llow, $E$ is the total internal energy and $P$ is the pressure. The mass fraction vector, is $\boldsymbol{i}=\left(f_{1}, f_{2}, f_{3}, f_{4}\right)^{T}$ and the column vectors $\boldsymbol{f}_{\mathbf{u}}$ and $f_{\mathrm{v}}$ are composed of the specific momentum of $i^{\text {th }}$ species

$$
\mathbf{f}_{\mathbf{u} i}=f_{i}\left(u+\tilde{u}_{i}\right), \quad \mathbf{f}_{\mathbf{v} i}=\tilde{f}_{i}\left(v+\tilde{v}_{i}\right)
$$

The velocity field $\left(\tilde{u}_{i}, \tilde{v}_{i}\right)$ of the $i^{t h}$ species is the drift velocity relative to the mean mixture velocity $(u, v)$ and is determined by

$$
\left(\tilde{u}_{i}, \tilde{v}_{i}\right)=\frac{\mu}{\rho S_{c}} \nabla f_{i}
$$

Here $\mu$ is the mixture dynamic viscosity to be determined in (2.11), and $S_{c}$ is the Schmidt number which is taken to be 0.22 . The viscous fluxes, $F_{v}$ and $G_{\nu}$ are given by

$$
\begin{aligned}
& F_{\nu}=\left(\begin{array}{c}
0 \\
\tau_{x x} \\
\tau_{y x} \\
u \tau_{x x}+v \tau_{y x}+\mu \frac{\bar{C}_{x}}{P_{x}} T_{x}-\rho \sum_{i=1}^{4} h_{i} \tilde{u}_{i} f_{i} \\
0
\end{array}\right), \\
& G_{\nu}=\left(\begin{array}{c}
0 \\
\tau_{x y} \\
\tau_{y y} \\
u \tau_{x y}+v \tau_{y y}+\mu \frac{\partial_{p}}{P_{r}} T_{y}-\rho \sum_{i=1}^{4} h_{i} \check{b}_{i} f_{i} \\
0
\end{array}\right) .
\end{aligned}
$$

where $0=(0,0,0,0)^{T}, T$ is the temperature, $\bar{C}_{p}$ is the mixture specific heat at constant pressure, $P_{r}$ is the Prandtl number (which is taken to be 0.72) for the normal air and $h_{i}$ is the specific enthalpy of the $i^{t h}$ species and given by

$$
h_{i}=h_{i}^{0}+\int_{0}^{T} C_{p_{i}}(s) d s
$$

where $h_{i}^{0}$ is the reference enthalpy of the $i^{\text {th }}$ species and the specific heat of the $i^{\text {th }}$ species at constant pressure, $C_{p_{i}}$ is represented as a fourth-order polynomial of $T$ (see [16]). The elements of the viscous stress tensor are given by

$$
\tau_{x_{i} x_{j}}=\mu\left(\frac{\partial u_{i}}{\partial x_{j}}+\frac{\partial u_{j}}{\partial x_{i}}\right)+\delta_{i j} \lambda \sum_{k=1}^{2} \frac{\partial u_{k}}{\partial x_{k}}
$$

where $\delta$ is the Kronecker delta symbol, and $\lambda$ is the bulk viscosity which is taken to be $-\frac{2}{3} \mu$ under the Stokes hypothesis. 
The equation of state is given by the assumption of the perfect gas law

$$
P=\rho \bar{R} T=R T \sum_{i=1}^{4} \rho f_{i} / M_{i}
$$

where $\bar{R}$ is a mixture gas constant with the universal gas constant $R$ and $M_{i}$ is the molecular weights of $i^{\text {th }}$ species. The energy $E$ is given by

$$
E=\rho \int_{0}^{T} \bar{C}_{p}(s) d s-P+\frac{1}{2} \rho\left(u^{2}+v^{2}\right)+\sum_{i=1}^{4} \rho f_{i} h_{i}^{0}
$$

where the mixture specific heat at constant pressure is given by

$$
\bar{C}_{p}=\sum_{i=1}^{4} C_{p_{i}} f_{i} / M_{i}
$$

2.1. The chemical models. We use the same models as in [7]. Each chemical species has different dynamical viscosity $\mu_{i}$ based on Sutherland's law and we obtain the mixture viscosity $\mu$ by Wilke's law [21], i.e.

$$
\begin{aligned}
\frac{\mu_{i}}{\mu_{0 i}} & =\left(\frac{T}{T_{0 i}}\right)^{3 / 2}\left(\frac{T_{0 i}+S_{i}}{T+S_{i}}\right), \\
\mu & =\sum_{i=1}^{4} \frac{\mu_{i} f_{i} / M_{i}}{\sum_{j=1}^{4} f_{j} / M_{j} \phi_{i j}}, \\
\phi_{i j} & =\frac{\left(1+\left[\left(\mu_{i} / \mu_{j}\right)\left(f_{j} / f_{i}\right)\right]^{\frac{3}{2}}\left(M_{i} / M_{j}\right)^{\frac{1}{4}}\right)^{2}}{\left[8\left(1+\left(M_{i} / M_{j}\right)\right)\right]^{\frac{1}{2}}} .
\end{aligned}
$$

Here $\mu_{0}, T_{0 i}$ and $S_{i}$ are constants. A modified Arrhenius Law gives the equilibrium reaction rate $k_{e}$, the forward reaction rate $k_{f}$ and the backward reaction rate $k_{b}$ as

$$
\begin{aligned}
& k_{e}=A_{e} T \exp \left(4.60517\left(E_{e} / T-2.915\right)\right) \\
& k_{f}=A_{f} \exp \left(-E_{f} /(D T)\right) \\
& k_{b}=k_{f} / k_{e}
\end{aligned}
$$

where the activation energy $E_{e}=12925, E_{f}=7200$ and the frequency factor $A_{e}=83.006156, A_{f}=5.541 \times$ $10^{14}$.

The species are ordered as follows: $\left(\mathrm{H}_{2}, \mathrm{O}_{2}, \mathrm{H}_{2} \mathrm{O}, \mathrm{N}_{2}\right)$, and the law of mass action is used to find the net rate of change in concentration of $i^{\text {th }}$ species $\dot{C}_{i}$ by the single reaction (2.1), i.e.

$$
\begin{aligned}
& \dot{C}_{1}=2\left(k_{f}\left[\mathrm{H}_{2}\right]^{2}\left[\mathrm{O}_{2}\right]-k_{b}\left[\mathrm{H}_{2} \mathrm{O}\right]^{2}\right) \\
& \dot{C}_{2}=-\left(k_{f}\left[\mathrm{H}_{2}\right]^{2}\left[\mathrm{O}_{2}\right]-k_{b}\left[\mathrm{H}_{2} \mathrm{O}\right]^{2}\right) \\
& \dot{C}_{3}=2\left(k_{f}\left[\mathrm{H}_{2}\right]^{2}\left[\mathrm{O}_{2}\right]-k_{b}\left[\mathrm{H}_{2} \mathrm{O}\right]^{2}\right)
\end{aligned}
$$

where [] denoted the net rate of change in concentration.

Finally, the chemical source term $C$ in (2.2) is given by

$$
C=\left(0,0,0,0, \dot{C}_{1} M_{1}, \dot{C}_{2} M_{2}, \dot{C}_{3} M_{3}, \dot{C}_{4} M_{4}\right)^{T},
$$

where $\dot{C}_{i}$ is the net rate of change in concentration of $i^{\text {th }}$ species by the reaction.

In Appendix C, a table of all the necessary coefficients and constants used for the reactive Navier-Stokes equations with species $\left(\mathrm{H}_{2}, \mathrm{O}_{2}, \mathrm{H}_{2} \mathrm{O}, \mathrm{N}_{2}\right)$ are given. 
3. The Multi-domain Spectral Method. In this section we describe the two crucial components of the Chebyshev multi domain code used in our work, i.e. the adaptive filtering and the penalty method for the stable interface conditions.

3.1. The adaptive filtering technique. It is well known that spectral methods may exhibit instabilities when applied to nonlinear equations. To stabilize the spectral scheme in an efficient way we use here filters to attenuate the high frequency modes of the function $q_{N}(x, t)$ smoothly to zero. Thus the filtered version of a polynomial $q_{N}$ is given by:

$$
q_{N}^{\sigma}(x, t)=\sum_{k=-N}^{N} \sigma(k / N) a_{k}(t) \phi_{k}(x),
$$

where $a_{k}$ is the transform coefficient and $\phi_{k}$ is the basis polynomial of order $k$ (generally the Fourier and Chebyshev polynomials for a periodic and non-periodic function respectively).

Following Vandeven [20] we define a filter function $\sigma(\omega)$ of order $p>1$ as a $C^{\infty}[-1,1]$ function satisfying

$$
\begin{aligned}
\sigma(0) & =1, \quad \sigma( \pm 1)=0, \\
\sigma^{(j)}(0) & =0, \quad \sigma^{(j)}( \pm 1)=0, \quad j \leq p
\end{aligned}
$$

where $\sigma^{(j)}$ denotes the $j$-th derivative.

It can be shown that the filtered sum (3.1) approximates the original function very well away from the discontinuities. A good example of filter function is the exponential filter. It is defined as

$$
\sigma(\omega)=\exp \left(-\alpha|\omega|^{\gamma}\right)
$$

where $-1 \leq \omega=k / N \leq 1, \alpha=-\ln \epsilon, \epsilon$ is the machine zero and $\gamma$ is the order of the filter.

The exponential filter offers the flexibility of changing the order of the filter simply by specifying a different $\gamma$. One does not have to write a different, filter for different order. Thus varying $\gamma$ with $N$ yields exponential accuracy according to [20]. In the present study the sixth order global smoothing $(\gamma=6)$ is used. If the order of the filter $\gamma$ is taken to be too small, say $\gamma \leq 4$, the method becomes too dissipative.

In the current application, the interaction of the aft cavity wall and the strong vortex generated by the shear layer flow over the cavity, creates large pressure variations near the comer of the aft cavity wall. The local sharp gradient can cause numerical instability and a heavier filter is needed to prevent the development of oscillations in this region. This heavy filtering can be used globally and maintain the stability of the scheme, however this dissipates out all fine scale structures, which is highly undesirable when the resolution of fine scale structures is essential for the understanding of the recessed cavity flameholder systems.

Since this is a local phenomenon, it is enough to apply a heavy filter only in points in this region. This Local Adaptive Filtering keeps the scheme stable, without dissipating fine scale features away from this region. The local adaptive filtering is carried out where conditions such as $q^{l} \leq q \leq q^{u}$ are violated. Here $q$ can be the mass fraction of each species $f_{i}$ and/or temperature $T$ and $q^{l}$ and $q^{u}$ denote the lower and upper tolerance limits of $q$. In this work, a filtering of the order $y=2$ or $\gamma=3$ is used to reduce the magnitude of the oscillations at those points.

The results of this work indicate that the local adaptive filtering is applied only in a few number (in the range of 1 to 7 ) of grid points around the corner of the aft wall once in a while.

3.2. Stable interface conditions. In this paper we use mainly the penalty type interface conditions, i.e. the boundary conditions are imposed only in a weak form $[10,11]$. Successful penalty interface conditions 
were constructed based on the characteristics for the Navier-Stokes equations in $[13,14,10]$ and for spectral method and for high-order finite difference methods in $[4,17,18]$, and a conservative form of penalty interface conditions was proposed [5] for the Legendre spectral method. Following the same idea as those works, we consider two interface conditions, i.e.

1. The averaging method, in which the interface conditions are obtained by averaging the state vectors of the two adjacent domains, and

2. The Penalty method in conservative form in which the interface conditions are satisfied only in a weak form, leaving the approximations not necessarily continuous at the interfaces.

In the following sections we will give the penalty interface conditions for the Euler and Navier-Stokes equations and also show that the averaging method is a subset of the penalty method.

3.2.1. Conservative penalty interface conditions. Consider equation 2.2 with the inviscid part, only, in the $x$-direction in the interval $-2 \leq x \leq 2$, i.e.,

$$
\frac{\partial q}{\partial t}+\frac{\partial F}{\partial x}=0
$$

For simplicity, assume that we have two domains in this interval with the interface at $x=0, q_{N}^{I}(x, t)$ denotes the numerical solution in the left domain $x \leq 0$ and $q_{M}^{I}(x, t)$ in the right domain $x \geq 0$. Note that the numerical solution is composed of two polynomials of different orders. The Legendre spectral penalty method is given by

$$
\begin{aligned}
\frac{\partial q_{N}^{I}}{\partial t}+\frac{\partial I_{N}^{I} F\left(q_{N}^{I}\right)}{\partial x}= & B\left(q_{N}^{I}(-2, t)\right)+ \\
& \tau_{1} Q_{N}(x)\left[f^{+}\left(q_{N}^{I}(0, t)\right)-f^{+}\left(q_{M}^{I I}(0, t)\right)\right]+ \\
& \tau_{2} Q_{N}(x)\left[f^{-}\left(q_{N}^{I}(0, t)\right)-f^{-}\left(q_{M}^{I}(0, t)\right)\right] \\
\frac{\partial q_{M}^{I I}}{\partial t}+\frac{\partial I_{M}^{I I} F\left(q_{M}^{I I}\right)}{\partial x}= & B\left(q_{M}^{I I}(2, t)\right)+ \\
& \tau_{3} Q_{M}(x)\left[f^{+}\left(q_{M}^{I I}(0, t)\right)-f^{+}\left(q_{N}^{I}(0, t)\right)\right]+ \\
& \tau_{4} Q_{M}(x)\left[f^{-}\left(q_{M}^{I I}(0, t)\right)-f^{-}\left(q_{N}^{I}(0, t)\right)\right]
\end{aligned}
$$

where $B$ is a boundary operator at the end points, i.e., $x= \pm 2$ and $I_{N}^{I}$ and $I_{M}^{I I}$ are the Legendre interpolation operators for the left and right domains respectively. . The positive and negative fluxes $f^{+}$and $f^{-}$are defined by

$$
f^{ \pm}=\int S \Lambda^{ \pm} S^{-1} d q
$$

with

$$
A \equiv \frac{\partial F}{\partial q}=S \Lambda S^{-1}
$$

The Jacobian matrix $A$ is assumed to be symmetric. $\Lambda^{+}$and $\Lambda^{-}$are the diagonal matrices composed of positive and negative eigenvalues of $A$ respectively. $Q_{N}(x)$ and $Q_{M}(x)$ are polynomials of orders $N$ and $M$ respectively such that they are zero at all the collocation points except the interface points $x=0$ (for example $Q_{N}(x)=\frac{(1-x / 2) T_{N}^{\prime}(x / 2)}{N^{2}}, 0 \leq x \leq 2$ where $T_{N}(x)$ is the Chebyshev polynomial of degree $\left.N\right)$. The penalty parameters $\tau_{1}, \tau_{2}, \tau_{3}$ and $\tau_{4}$ are all constants. Since we are interested only in the interface conditions, we ignore the boundary operator $B$ at $x= \pm 2$. Define the discrete scalar product $(p, q)_{N}=\sum_{i=0}^{N} p^{T}\left(\xi_{i}\right) q\left(\xi_{i}\right) \omega_{i}$. $\omega_{i}$ is the weight in the Gauss-Lobatto-Legendre quadrature formula. With the discrete product, the energy 
$E(t)$ is defined by $E(t)=\left(q_{N}^{I}(x, t), q_{N}^{I}(x, t)\right)_{N}+\left(q_{M}^{I I}(x, t), q_{M}^{I I}(x, t)\right)_{M}$. The stability conditions of penalty parameters are given by the following theorem [5]:

THEOREM 3.1. The energy is bounded by the initial energy of the system if the following conditions are satisfied;

$$
\begin{aligned}
& 2 \omega_{N}^{I} \tau_{1} \leq 1, \quad 2 \omega_{N}^{I} \tau_{2} \geq 1, \quad 2 \omega_{M}^{I I} \tau_{3} \leq-1, \quad 2 \omega_{M}^{I I} \tau_{4} \geq-1, \\
& \omega_{N}^{I} \tau_{1}-\omega_{M}^{I I} \tau_{3}=1, \quad \omega_{N}^{I} \tau_{2}-\omega_{M}^{I I} \tau_{4}=1 .
\end{aligned}
$$

3.2.2. The penalty method for the Euler Equations. The penalty method in the case of the 2-D Euler equation is given by

$$
\begin{aligned}
\frac{\partial q_{N}}{\partial t}+\frac{\partial I_{N} F\left(q_{N}\right)}{\partial x}+\frac{\partial I_{N} G\left(q_{N}\right)}{\partial y}= & \tau_{1,3} Q(x, y)\left[f^{+}\left(q_{N}\right)-f^{+}\left(q_{M-}\right)\right]+ \\
& \tau_{2,4} Q(x, y)\left[f^{-}\left(q_{N}\right)-f^{-}\left(q_{M-}\right)\right]
\end{aligned}
$$

where $q_{M-\text { is }}$ is the state vector of the adjacent domain at the interface of degree $M, \tau_{1,3}\left(\tau_{2,4}\right)$ denotes $\tau_{1}\left(\tau_{2}\right)$ and $\tau_{3}\left(\tau_{4}\right)$ respectively. $\tau_{1}$ and $\tau_{2}\left(\tau_{3}\right.$ and $\left.\tau_{4}\right)$ are the penalty parameters for the right(left) in $x$-direction and top(bottom) in $y$-direction respectively. $Q(x, y)$ is a polynomial which vanishes at all of interior points of the domain and is equal to 1 at the four interfaces. Note that the boundary operator $B$ does not appear in the scheme. Let $A$ be the linearized Jacobian matrix (around a state vector $q_{0}$ ) of two inviscid fluxes

$$
A=\left.\left(\frac{\partial F}{\partial q}, \frac{\partial G}{\partial q}\right) \cdot \vec{n}\right|_{q_{0}} .
$$

where $\vec{n}=\left(n_{x}, n_{y}\right)$ is the unit outward normal vector. Since the matrix $A$ is symmetric, there exists $S$ such that

$$
A=S \Lambda S^{-1}
$$

where $\Lambda$ is a diagonal matrix composed of eigenvalues of $A$. Then $A=A^{+}+A^{-}$and $A^{ \pm}=S A^{ \pm} S^{-1} \cdot \Lambda^{ \pm}$is defined as in previous section. Splitting $A$ yields

$$
f^{ \pm}=A^{t} q_{0}
$$

where $f^{ \pm}$is obtained from the linearized state.

REMARK 1. Since $\vec{n}=\left(n_{x}, n_{y}\right)$ is taken to be outward normal vector, the stability condition (3.8) is now modified and given as

$$
\begin{aligned}
& 2 \omega_{N}^{I} \tau_{1} \leq 1, \quad 2 \omega_{N}^{I} \tau_{2} \geq 1, \quad 2 \omega_{M}^{I I} \tau_{3} \leq 1, \quad 2 \omega_{M}^{I I} \tau_{4} \geq 1, \\
& \omega_{N}^{I} \tau_{1}+\omega_{M}^{I I} \tau_{4}=1, \quad \omega_{N}^{I} \tau_{2}+\omega_{M}^{I I} \tau_{3}=1 .
\end{aligned}
$$

The Jacobian matrix $A$ and its eigenvalue matrix $\Lambda$ are given in Appendix $A$.

For illustration, we consider the propagation of a Gaussian density peak at the center of rectangular physical domains. The physical domain is partitioned with 16 sub-domains. The interface conditions between the domains are imposed according to the penalty Euler equations as discussed above. Characteristic boundary conditions are imposed at the outer physical boundaries. The results presented in figure 3.1 indicate that the penalty formulation works well. From the numerical experiments of this problem, we observe 

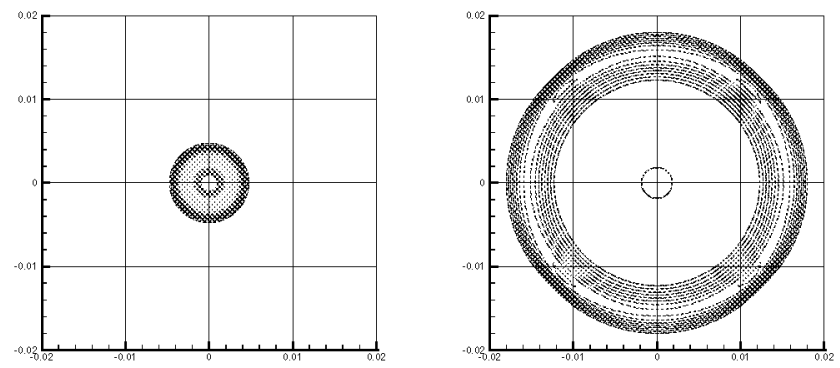

FIG. 3.1. The propagation of a density peak with the penalty Euler equations with 16 sub-domains: The initial condition (aft) and the solution (right) at $t=0.03604 \mathrm{~ms}$ are given.

that reflections can be created at the interface across the adjacent domains depending on the choice of the penalty parameters. Thus proper choice of the penalty parameters should take into account reflections from the interfaces. To demonstrate the above formulation for the Euler Equations, We will return to this issue in a future paper ${ }^{1}$.

3.2.3. The penalty method for the Navier-Stokes Equations. When dealing with the Navier Stokes equation, we keep the penalty form for the Euler fluxes and add a penalty term for the viscous fluxes. The stability of this procedure stems from the fact that the Jacobian matrices for the full reactive Navier-Stokes equation can be symmetrized by the same similarity transformation (see Appendix B). Thus we get the system:

$$
\begin{aligned}
\frac{\partial q_{N}}{\partial t}+\frac{\partial I_{N} F}{\partial x}+\frac{\partial I_{N} G}{\partial y}= & \frac{\partial I_{N} F_{\nu}}{\partial x}+\frac{\partial I_{N} G_{\nu}}{\partial y}+ \\
& \tau_{1,3} Q(x, y)\left[f^{+}\left(q_{N}\right)-f^{+}\left(q_{M-}\right)\right]+ \\
& \tau_{2,4} Q(x, y)\left[f^{-}\left(q_{N}\right)-f^{-}\left(q_{M-}\right)\right]+ \\
& \tau_{6,8} Q(x, y)\left[\mathbf{A}_{\nu} \cdot \mathbf{q}_{N}-\mathbf{A}_{\nu} \cdot \mathbf{q}_{M-}\right]+ \\
& \tau_{5,7} Q(x, y)\left[\mathbf{A}_{\nu} \cdot \partial \mathbf{q}_{N}-\mathbf{A}_{\nu} \cdot \partial \mathbf{q}_{M-}\right]
\end{aligned}
$$

Here $f^{ \pm}$are same as defined in the previous section and the Jacobian matrix vector $\mathbf{A}_{\nu}$ is given by

$$
\mathbf{A}_{\nu}=\left.\left(\frac{\partial F_{\nu}}{\partial q_{x}} n_{x}, \frac{\partial G_{\nu}}{\partial q_{y}} n_{y}\right)\right|_{q_{0}}
$$

and

$$
\mathbf{q}=(q, q), \quad \partial \mathbf{q}=\left(q_{x}, q_{y}\right),
$$

where again $q_{--}$and $\partial q_{-}$- denote the adjacent domains state vectors and their derivatives. Note that the penalty terms $\mathbf{A}_{\nu} \cdot \partial \mathbf{q}$ does not appear in $[4,17,18]$. The penalty parameters $\tau_{5,7}$ and $\tau_{6,8}$ are defined in the same way as in the previous section. To seek stable penalty parameters we split the inviscid and viscous fluxes and keep the stability conditions of $\tau_{1,2,3,4}$ for the inviscid fux as in Theorem 3.1. The stability conditions of $\tau_{5,7}$ and $\tau_{6,8}$ are given in the following Theorem :

\footnotetext{
1. H. Jung, PhD thesis, Div. of Applied Math., Brown University, 2002
} 
THEOREM 3.2. The penalty method for the Navier-Stokes equations (3.14) is stable if the penalty parameters $\tau_{j}, j=1 . .4$ are as in Theorem 3.1 and the rest satisfy.

$$
\begin{aligned}
& \omega_{N} \tau_{6} \leq 0 \\
& \omega_{N} \tau_{6}-\omega_{M} \tau_{8}=0 \\
& 1+\omega_{N} \tau_{5}-\omega_{M} \tau_{7}=0 \\
&\left(\frac{1}{\omega_{M}}+\frac{1}{\omega_{N}}\right) \omega_{M}^{2} \tau_{7}^{2}-2 \tau_{7}+4 \omega_{N} \tau_{6}+\frac{1}{\omega_{M}} \leq 0
\end{aligned}
$$

Proof.

As in the proof of Theorem 3.1, we assume that we have two domains and by multiplying the equations by the state vectors, we get

$$
\frac{1}{2} \frac{d}{d t} E(t) \leq[\text { Inviscid }]+[\text { Viscous }]
$$

where [Inviscid] and [Viscous] denote the terms from inviscid and viscous parts of the equation respectively. The conditions for $\tau_{1,2}$ and $\tau_{3,4}$ given in the Theorem 3.1 assure that the first term [Inviscid] is negative. The [Viscous] part at the interface is given by

$$
\begin{aligned}
\text { [Viscous }]= & q^{T} A_{\nu} q^{\prime}-\sum_{i=0}^{N} q_{i}^{\prime T} A_{\nu} q_{i}^{\prime} \omega_{i}- \\
& q^{T} A_{\nu} q_{-}^{\prime}-\sum_{j=0}^{M} q_{i-}^{\prime} A_{\nu} q_{i-}^{\prime} \omega_{j}+ \\
& \tau_{5} \omega_{N}\left[q^{T} A_{\nu} q^{\prime}-q^{T} A_{\nu} q_{--}^{\prime}\right]+\tau_{\gamma} \omega_{M}\left[q_{--}^{T} A_{\nu} q_{-}^{\prime}-q_{-}^{T} A_{\nu} q^{\prime}\right]+ \\
& \tau_{6} \omega_{N}\left[q^{T} A_{\nu} q-q^{T} A_{\nu} q_{-}\right]+\tau_{g} \omega_{M}\left[q_{-}^{T} A_{\nu} q_{-}-q_{-}^{T} A_{\nu} q\right]
\end{aligned}
$$

where $q^{\prime}$ denotes the derivative of $q$ either in $x$ or $y$ direction, $\omega$ is the Legendre weight, and $A_{\nu}$ is

$$
A_{1}=\left.\left(\frac{\partial F_{\nu}}{\partial q_{x}}, \frac{\partial G_{\nu}}{\partial q_{y}}\right) \cdot \vec{n}\right|_{q_{0}} .
$$

Since all the eigenvalues of $A_{y}$ are non-negative, every term inside the summations in the above equation is not negative, and we would like to keep the boundary terms. Thus we get the energy estimate such as

$$
\begin{aligned}
\frac{1}{2} \frac{d}{d t} E(t) \leq & {\left[q^{T} A_{\nu} q^{\prime}-\omega_{N} q^{T} A_{\nu} q^{\prime}-q_{-}^{T} A_{\nu} q_{-}^{\prime}-\omega_{M} q_{-}^{T} A_{\nu} q_{-}^{\prime}\right]+} \\
& \tau_{5} \omega_{N}\left[q^{T} A_{\nu} q^{\prime}-q^{T} A_{\nu} q_{--}^{\prime}\right]+\tau_{7} \omega_{M}\left[q_{-}^{T} A_{\nu} q_{--}^{\prime}-q_{-}^{T} A_{\nu} q^{\prime}\right]+ \\
& \tau_{6} \omega_{N}\left[q^{T} A_{\nu} q-q^{T} A_{\nu} q_{-}\right]+\tau_{8} \omega_{M}\left[q_{-\ldots}^{T} A_{\nu} q_{-}-q_{\ldots}^{T} A_{\nu} q\right] .
\end{aligned}
$$

The RHS of (3.21) can be rewritten as

$$
\text { RHS }=u^{T} B u
$$

where $u=\left(q, q_{-}, q^{\prime}, q_{-}^{\prime}\right)$ and $B$ is given by

$$
B=\left(\begin{array}{rrrr}
2 \sigma_{6} A_{\nu} & -\sigma_{6} A_{\nu}-\sigma_{8} A_{\nu}^{T} & \left(1+\sigma_{5}\right) A_{\nu} & -\sigma_{5} A_{\nu} \\
-\sigma_{6} A_{\nu}^{T}-\sigma_{8} A_{\nu} & 2 \sigma_{3} A_{\nu^{\prime}} & -\sigma_{7} A_{\nu} & \left(-1+\sigma_{7}\right) A_{\nu} \\
\left(1+\sigma_{5}\right) A_{\nu}^{T} & -\sigma_{7} A_{\nu}^{T} & -2 \omega_{N} A_{\nu} & 0 \\
-\sigma_{5} A_{\nu}^{T} & \left(-1+\sigma_{7}\right) A_{\nu}^{T} & 0 & -2 \omega_{M} A_{\nu}
\end{array}\right)
$$


with $0=\operatorname{diag}(0,0,0,0), \sigma_{5}=\omega_{N} \tau_{5}, \sigma_{6}=\omega_{N} \tau_{6}, \sigma_{7}=\omega_{M} \tau_{7}$ and $\sigma_{8}=\omega_{M} \tau_{8}$. It is sufficient for the proof if $B$ can be shown to be negative semi-definite. This first leads to:

$$
\omega_{N} \tau_{6} \leq 0, \quad \omega_{N} \tau_{6}=\omega_{M} \tau_{8}, \quad 1+\omega_{N} \tau_{5}-\omega_{M} \tau_{7}=0
$$

Note that we use here the fact that $A_{\nu}$ is symmetrizable (see Appendix B). Taking into account (3.24), $B$ becomes

$$
B=\left(\begin{array}{ccc}
2 \sigma_{6} & -\sigma_{7} & -1+\sigma_{7} \\
-\sigma_{7} & -2 \omega_{N} & 0 \\
-1+\sigma_{7} & 0 & -2 \omega_{M}
\end{array}\right)
$$

To ensure negative semi-definiteness, $\operatorname{det}(B) \leq 0$ and therefore

$$
\left(\frac{1}{\omega_{M}}+\frac{1}{\omega_{N}}\right) \sigma_{7}^{2}-2 \frac{1}{\omega_{M}} \sigma_{7}+4 \sigma_{6}+\frac{1}{\omega_{M}} \leq 0
$$

Thus

$$
\sigma_{7}^{-} \leq \sigma_{7} \leq \sigma_{7}^{+}
$$

where

$$
\sigma_{7}^{ \pm}=\frac{\omega_{N}}{\omega_{M}+\omega_{N}} \pm \sqrt{\frac{\left(\omega_{N} \omega_{M}\right)\left(1-4 \sigma_{6}\left(\omega_{M}+\omega_{N}\right)\right)}{\left(\omega_{N}+\omega_{M}\right)^{2}}}
$$

Here we note that the condition that $\sigma_{6} \leq \frac{-1}{4\left(\omega_{N}+\omega_{M}\right)}$ must be also satisfied in order for $\sigma_{7}$ to have real root. This yields the conditions in the Theorem. $\square$ Note that these conditions are given independently of the local flow properties. And moreover, the penalty parameters of each domain are constrained by its adjacent domain.

REMARK 2. For $\vec{n}$ to be outward normal vector the condition (3.17) is now given by

$$
\begin{gathered}
\omega_{N} \tau_{6} \leq 0, \quad \omega_{N} \tau_{6}+\omega_{M} \tau_{8}=0, \quad 1+\omega_{N} \tau_{5}+\omega_{M} \tau_{7}=0 \\
\left(\frac{1}{\omega_{M}}+\frac{1}{\omega_{N}}\right) \omega_{M}^{2} \tau_{7}^{2}+2 \tau_{7}+4 \omega_{N} \tau_{6}+\frac{1}{\omega_{M}} \leq 0
\end{gathered}
$$

with the conditions (3.13)

3.2.4. The averaging method. We show in this Section that the averaging method can also be written as a penalty method with a particular choice of the parameters.

Euler Equations : We start first with the Euler equations: consider the following penalty method:

$$
\begin{aligned}
\frac{\partial q}{\partial t}+\frac{\partial F}{\partial x}+\frac{\partial G}{\partial y}= & \tau_{1,3} Q(x, y)\left[f^{+}(q)-f^{+}(q-)\right]+ \\
& \tau_{2,4} Q(x, y)\left[f^{\prime-}(q)-f^{\prime-}\left(q_{-}\right)\right]
\end{aligned}
$$

where

$$
f^{\prime t}=\left.\left(A^{ \pm} q_{x}, A^{t} q_{y}\right) \cdot \vec{n}\right|_{q_{0}}
$$

Note that the penalty terms use the derivative of the fluxes. 
THEOREM 3.3. If $\tau_{1}=\tau_{3}=\frac{1}{2}, \tau_{2}=\tau_{4}=\frac{1}{2}$ then the above penalty method (3.29) is equivalent to the averaging method and is stable.

Proof. We prove the theorem at the interface $x=0$ with the rectangular domain and assume that, $N=M$. If $\tau_{1}=\tau_{3}=\frac{1}{2}$, and $\tau_{2}=\tau_{4}=\frac{1}{2}$ then the method becomes

$$
\left.\frac{\partial q^{I}}{\partial t}\right|_{x=0}=\left.\frac{\partial q^{I I}}{\partial t}\right|_{x=0}=-\frac{1}{2}\left(\frac{\partial F^{I}}{\partial x}+\frac{\partial F^{I I}}{\partial x}\right)-\frac{\partial G}{\partial y}
$$

and this is obviously equivalent to the Averaging Method. Here note that $\frac{\partial G^{I}}{\partial y}=\frac{\partial G^{I I}}{\partial y}=\frac{\partial G}{\partial y}$. Following the same procedure in Theorem 3.2, the energy equation becomes

$$
\begin{aligned}
\frac{1}{2 \omega_{N}} \frac{d}{d t} E(t)= & -\left.\frac{1}{2 \omega_{N}}\left(q^{I} A q^{I}-q^{I I} A q^{I}\right)\right|_{0}+\left.\left(\tau_{1} q^{I}-\tau_{3} q^{I I}\right) A^{+}\left(q_{x}^{I}-q_{x}^{I I}\right)\right|_{0} \\
& +\left.\left(\tau_{2} q^{I}-\tau_{4} q^{I I}\right) A^{-}\left(q_{x}^{I}-q_{x}^{I I}\right)\right|_{0} .
\end{aligned}
$$

Since $\tau_{1}=\tau_{3}=\frac{1}{2}, \tau_{2}=\tau_{4}=\frac{1}{2}$, and $q^{I}(0, y, t)=q^{I}(0, y, t)$, the RHS of the above equation vanishes and the energy is bounded by the initial energy.

The Navier-Stokes Equations : The averaging method for the N-S equations can be presented as

$$
\begin{aligned}
\frac{\partial q}{\partial t}+\frac{\partial F}{\partial x}+\frac{\partial G}{\partial y}= & \frac{\partial F_{\nu^{\prime}}}{\partial x}+\frac{\partial G_{\nu}}{\partial y}+ \\
& \tau_{1,3} Q(x, y)\left[f^{\prime+}(q)-f^{\prime+}\left(q_{-}\right)\right]+ \\
& \tau_{2,4} Q(x, y)\left[f^{\prime--}(q)-f^{\prime--}\left(q_{-}\right)\right]+ \\
& \tau_{5,7} Q(x, y)\left[\mathbf{A}_{\nu} \cdot \partial^{2} \mathbf{q}_{-}-\mathbf{A}_{\nu} \cdot \partial^{2} \mathbf{q}_{-}\right]+ \\
& \tau_{6,8} Q(x, y)\left[\mathbf{A}_{\nu} \cdot \partial \mathbf{q}-\mathbf{A}_{\nu} \cdot \partial \mathbf{q}_{--}\right]
\end{aligned}
$$

where $\partial^{2} q$ is the second derivative of $q$ in either $x$ or $y$ direction.

THEOREM 3.4. If $\tau_{1}=\tau_{3}=\frac{1}{2}, \tau_{2}=\tau_{4}=\frac{1}{2}, \tau_{5}=\tau_{7}=\frac{1}{2}$, and $\tau_{6}=-\tau_{8}=-\frac{1}{2 \omega_{N}}$, then the approximation is continuous at the interface and the scheme (3.33) is stable.

Proof. If $\tau_{1}=\tau_{3}=\frac{1}{2}, \tau_{2}=\tau_{4}=\frac{1}{2}, \tau_{5}=\tau_{7}=\frac{1}{2}$, and $\tau_{3}=-\tau_{8}=-\frac{1}{\omega_{N}}$, then (3.33) becomes,

$$
\begin{aligned}
\left.\frac{\partial q^{I}}{\partial t}\right|_{x=0}=\left.\frac{\partial q^{I I}}{\partial t}\right|_{x=0}= & -\frac{1}{2}\left(\frac{\partial F^{I}}{\partial x}+\frac{\partial F^{I I}}{\partial x}\right)-\frac{\partial G}{\partial y} \\
& +\frac{1}{2}\left(\frac{\partial F_{\nu}^{I}}{\partial x}+\frac{\partial F_{\nu}^{I I}}{\partial x}\right)+\frac{\partial G_{\nu}}{\partial y} \\
& +\frac{1}{\omega_{N}} \mathbf{A}_{\nu} \cdot\left(\partial \mathbf{q}^{I I}-\partial \mathbf{q}^{I}\right),
\end{aligned}
$$

and this ensures the continuity of the approximation at the interface. If the approximation is smooth enough such that the derivative of $q$ is continuous at the interface then this becomes the averaging method.

Thus we get for the energy:

$$
\begin{aligned}
\frac{1}{2 \omega_{N}} \frac{d}{d t} E(t)= & -\left.\frac{1}{2 \omega_{N}}\left(q^{I} A q^{I}-q^{I I} A q^{I I}-2 q^{I} A_{\nu} q_{x}^{I}+2 q^{I I} A_{\nu} q_{x}^{I I}\right)\right|_{x=0}- \\
& \int_{-2}^{0} q_{x}^{I} A_{\nu} q_{x}^{I} d x-\int_{0}^{2} q_{x}^{I I} A_{\nu} q_{x}^{I I} d x+ \\
& {\left.\left[\left(\tau_{1} q^{I}-\tau_{3} q^{I}\right) A^{+}+\left(\tau_{2} q^{I}-\tau_{4} q^{I}\right) A^{-}\right]\left(q_{x}^{I}-q_{x}^{I I}\right)\right|_{x=0}+} \\
& {\left.\left[\left(\tau_{5} q^{I}-\tau_{\tau} q^{I I}\right) A_{\nu}\left(q_{x x}^{I}-q_{x x}^{I I}\right)+\left(\tau_{6} q^{I}-\tau_{8} q^{I I}\right) A_{\nu}\left(q_{x}^{I}-q_{x}^{I I}\right)\right]\right|_{x=0} }
\end{aligned}
$$


Since $q^{I}(0, y, t)=q^{I I}(0, y, t)$, we have

$$
\begin{aligned}
\frac{1}{2 \omega_{N}} \frac{d}{d t} E(t) \leq & q^{I}\left(\left[\left(\tau_{1}-\tau_{3}\right) A^{+}+\left(\tau_{2}-\tau_{1}\right) A^{-}\right]\left(q_{x}^{I}-q_{x}^{I I}\right)+\right. \\
& \left(\tau_{5}-\tau_{7}\right) A_{\nu}\left(q_{x x}^{I}-q_{x x}^{I I}\right) \\
& \left.\left(\tau_{6}-\tau_{8}+\frac{1}{\omega_{N}}\right) A_{\nu}\left(q_{x}^{I}-q_{x}^{I I}\right)\right)\left.\right|_{x=0} .
\end{aligned}
$$

Thus if $\tau_{1}=\tau_{3}=\frac{1}{2}, \tau_{2}=\tau_{4}=\frac{1}{2}, \tau_{5}=\tau_{7}=\frac{1}{2}$, and $\tau_{6}=-\tau_{8}=-\frac{1}{2 \omega_{N}}$, the RHS vanishes.

3.2.5. Adaptive averaging. To ensure the stability of the scheme at some particular collocation points where the solution become singular and unstable, we use the averaging method adaptively at selective grid points. In particular, we switched from the penalty method to the averaging when the following criteria was satisfied:

$$
\max \left(\frac{\left|\rho-\rho_{-}\right|}{\left|\rho+\rho_{-}\right|}, \frac{\left|T-T_{-}\right|}{\left|T+T_{-}\right|}\right) \geq C_{\text {ave }}
$$

or

$$
\frac{\left|P-P_{-}\right|}{\left|P+P_{-}\right|} \geq C_{\text {ave }}
$$

where $C_{\text {ave }}$ is a non-negative constant. Note that $C_{a v e}=0$ leads to the averaging method, whereas a large $C_{a v e}$ results in the penalty method. For the value of $C_{\text {ave }}$ used in this paper, we found out that there were very few points in which one needs to switch from the penalty to the averaging procedure. Moreover this happened only at very few time steps.

4. The Cavity System And Numerical Configurations. In this section we describe the set up of the simulations of the recessed cavity flameholders by the spectral multi-domain technique presented above. The main goal of this experiment is to investigate how the geometry of the aft wall affects the flame stability.

4.1. Physical setup. In the SCRAMJet community, a cavity with the length-to-depth ratio $L / D<$ $7 \sim 10$ is usually categorized as an 'open' cavity since the upper shear layer re-attaches at the back face [2]. In this work, we choose the $L / D$ of the baseline cavity to be 4 and thus the open cavity system is considered. The coordinates of the cavity are $(7 \mathrm{~cm},-1 \mathrm{~cm})$ for the upper left and $(11 \mathrm{~cm},-2 \mathrm{~cm})$ for the right bottom corners of cavity. With the length of the neck of the cavity fixed to be $4 \mathrm{~cm}$, we consider three different angles of the right corner of the floor of the cavity $(60,45$ and 30), we then compare each one with the case of the rectangular aft wall. The fluid conditions are given as followings; the free stream Mach number $M=1.91$, total pressure $P=2.82(\mathrm{~atm})$, total temperature $T=830.6(\mathrm{~K})$ and normalized Reynolds number $R_{e}=3.9 \times 10^{7}(1 / \mathrm{m})$. Note that the Reynolds number is here normalized and has a unit of $1 /$ [length], also the Reynolds number based on the cavity dimensions is $O\left(10^{5}\right)$. The boundary layer thickness scale is $\delta=5 \times 10^{-4}(\mathrm{~m})$, and finally, the wall temperature is $T_{w}=460.7835(K)$. The initial configuration for the baseline cavity system is shown in Figure 4.1.

4.2. Numerical setup. We have conducted two different experiments for each of the following cases (1) non-reacting cold flow and (2) reacting flow. We use 9 and 17 subdomains for both cases 1 and 2. For the outflow conditions at the exit of the system and at the upper boundary, we mainly use a semi-infinite mapping in order to reduce the possible reflections at the boundaries. The characteristic boundary conditions are also applied and will be discussed in the next section and compared to the mapping. For the case of the reactive flows, the cavity was initially filled with Hydrogen fuel with fuel-to-total gas ratio of 0.5 . The order 


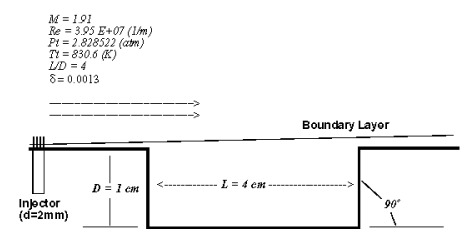

Fig. 4.1. The initial configuration for the baseline canity system.

of the polynomial of approximation in $y$ direction in the domain beside the wall is taken large enough to resolve the boundary layer well. Finally the adaptive filtering is turned on if the mass fraction of Hydrogen and Oxygen exceed the range of $-0.09 \leq f_{\mathrm{I}_{2}} \leq 1.09,-0.02 \leq f_{\mathrm{O}_{2}} \leq 0.25$ and the temperature exceeds the range of $300(K) \leq T \leq 3500(K)$. As the shear layer and the complex features of the flows develop, the adaptivity criteria for applying the local smoothing is satisfied at some points. In the calculations, we use the $3 \mathrm{rd}$ and $2 \mathrm{nd}$ order local filtering for the non-reactive and reactive flows respectively. It turns out that the local smoothing was applied in very few points at the upper comer of the cavity wall.

For the adaptive averaging, we use the criteria constant $C_{\text {ave }}$ such that the difference of the state vectors (or pressure) between the two adjacent domains is less than 10\%. In figure 4.2 the Penalty Navier-Stokes equations were considered for the non-reactive cold flows. As evident from the contours of the density, the approximations were well matched at the interfaces. Here the outer boundary was approximated by using the characteristic conditions of the inviscid fluxes. The adaptive averaging, with the given adaptivity conditions above, took place at only a few points. The characteristic boundary conditions using the inviscid fluxes yield good results for both the problems of the density peak propagation and the non-reactive cold flows. As in figure 1, we observe that there exist penalty parameters satisfying the stability conditions that may induce reflecting modes at the interfaces.

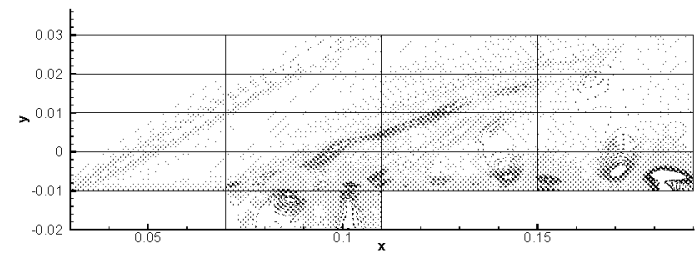

FIG. 4.2. The non-reactive cold flows with the penalty Navier-Stokes equations: the density contours are given in this figure at $t=0.25 \mathrm{~ms}$. 17 domains are used and the boundaries of each domain are shown.

\section{Results And Discussion.}

5.1. Pressure history. Figure 5.1 shows the pressure history of the non-reactive cold flows for the various angles of the aft wall at two different locations inside the cavity, i.e. at the center, $(x, y)=$ $(8.5 \mathrm{~cm},-1.5 \mathrm{~cm})$, and at the middle of the floor $(x, y)=(8.5 \mathrm{~cm},-1.9 \mathrm{~cm})$.

These figures show that the pressure fluctuations in cavities with lower angle of the aft are weaker than in cavities with higher angles. It is also shown that the attenuation of the pressure fluctuations are obtained both at the center and the middle of the floor of the cavity. It is interesting to observe that the patterns of the pressure fuctuations for a given angle at different locations are different depending on the angle. In the 

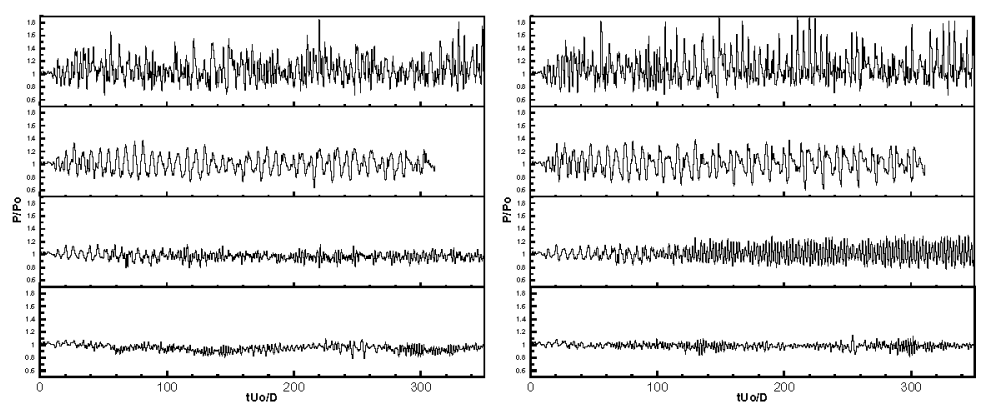

FIG. 5.1. Pressure history for non-reactive flows: the left panel represents the pressure history at the center of the cavity and the right panel at the middle of the floor of the cavity. Each panel shows the case of $90,60,45$ and 30 degree cavity walls from top to bottom.
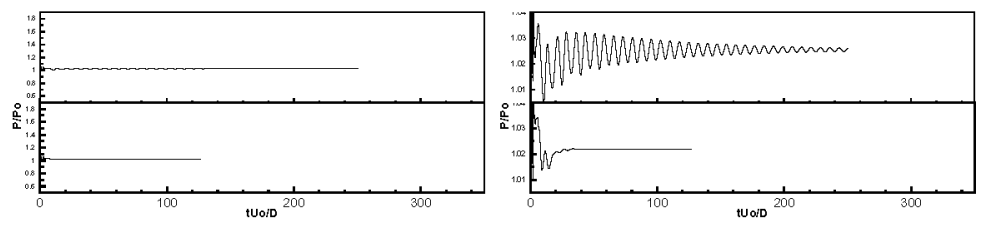

FIG. 5.2. Pressure history of the non-reactive flows with the use of the th order filter: the left parel represents the pressure history at the center of cavity and the right panel shows the left panel in a smaller scale. Each panel shows the case of 90 , and 30 degree cavity walls from top to boltom. Note that the scale of the right parel is different from the left.

case of the 30 degree aft wall, the pressure fluctuations are almost the same at the two locations considered whereas the case of 45 degree shows a difference in the patterns of the pressure fluctuations between the two locations. The pressure fluctuations at the bottom grows greater than that at the center after some time.
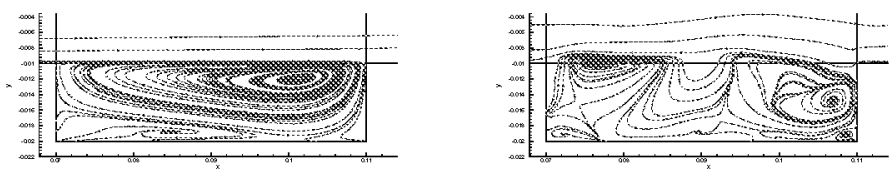

FIG. 5.3. Streamlines: the left figure shows the streamlines at $t=1.685 \mathrm{~ms}$ for the global fittering order $\gamma=4$ and the right at $t=2.38 \mathrm{~ms}$ for $\gamma=6$.

Figure 5.2 shows the pressure history when the heavy global filter is applied (in this case, the 4 th order filter was used). Unlike the previous case illustrated in Figure 5.1, where the 6th order global filter is used, the pressure fluctuations eventually decay out and a large recirculation zone is formed inside the cavity without any severe pressure fluctuations. Note that the scale in the left panel shown is the same as in Figure 5.1 while the right panel is shown in a smaller scale for a closer look. This figure shows that the large recirculation zone(s) formed inside the cavity obtained by the lower order numerical scheme is induced not physically but rather artificially due to the heavy numerical dissipations. This is clearly shown in Figure 5.3. In this figure a large recirculation zone is observed - this zone is formed earlier than this streamlines are captured - when the 4 th order filter is used(left figure) and an almost steady state is already reached as the pressure history indicates in Figure 5.2. We find from the numerical results that the large recirculation is very stable once it forms. This large recirculation and the steady state solutions are not observed in the case of $\gamma=6$ (right). For the case of $\gamma=6$ instead of the large single recirculation zone, smaller scale vortex 
circulations are formed and they are interacting with each other, never reaching the steady state with time. This result shows that for these sensitive problems, high order accuracy should be used in order to minimize the effect of the numerical dissipation.

Figure 5.4 shows the case of the reactive flows for the 90 and 30 degree aft walls. Similar features of the pressure fluctuations are shown as in the non-reactive flows. However the pressure fluctuations are much more attenuated for both the 90 and 30 degree walls than in the non-reactive cold flows. In the reactive cases Hydrogen fuel, which was initially supplied inside the cavity was consumed. As time elapses, the fuel is consumed out with the production of the water for these cases.
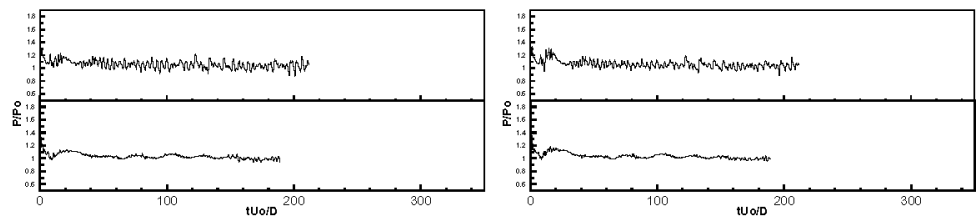

FIG. 5.4. Pressure history for reactive flows: the left panel represents the pressure history at the center of cavity and the right panel at the middle of the floor of cavity. Each panel shows the case of 90 and 30 degree cavity walls from top to bottom.
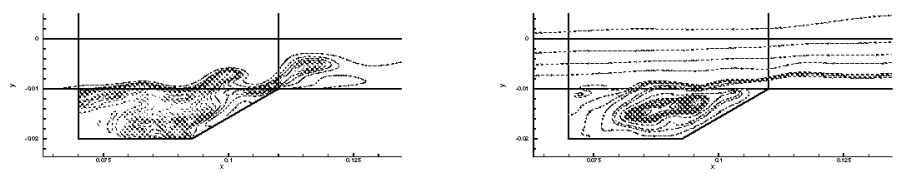

FIG. 5.5. The water contour of the reactive flows: the left the water density contour is given in the left figure and its streamlines in the right figure at $t=0.135 \mathrm{~ms}$.

These results demonstrate that simulations of cold flows do not necessarily shed light on the behavior of reactive flows.

\subsection{Flow fields.}

\section{Non-reactive cold flow}

Figure 5.6 shows the density contours and streamlines for the $90,60,45$ and 30 degree walls at the instant time $t=2.4 \mathrm{~ms}$. As shown in the figure, the shear layer is becoming weaker as the degree of angle of the aft wall and the flow fields are becoming more regularized for the case of the lower angle. And note that the density compression at the corner of the aft wall is also becoming weaker for the more slanted wall cases.

Figure 5.8 shows the streamlines corresponding to the each case of Figure 5.7. Note that compared to the non-reactive cases, the shear layers are less developed for the reactive cases. As the figures of the pressure fluctuation history and Figure 5.8 indicate, the shear lavers are weak for both the 90 and the 30 degree walls in the reactive cases.

\section{Reactive flow}

Figure 5.7 shows the water contour inside the cavity for the different angles at different time. Here we define the region where the flames are generated to be same as the region where the water is produced. As the Hydrogen fuel is consumed, the water is produced and starts to be expelled from the cavity to the main channel. The flame-holding efficiency is enhanced if the chemical radicals (water in this case) are stably circulating and long lasting before they are expelled from the cavity. Figure 5.7 shows that the lower angled 

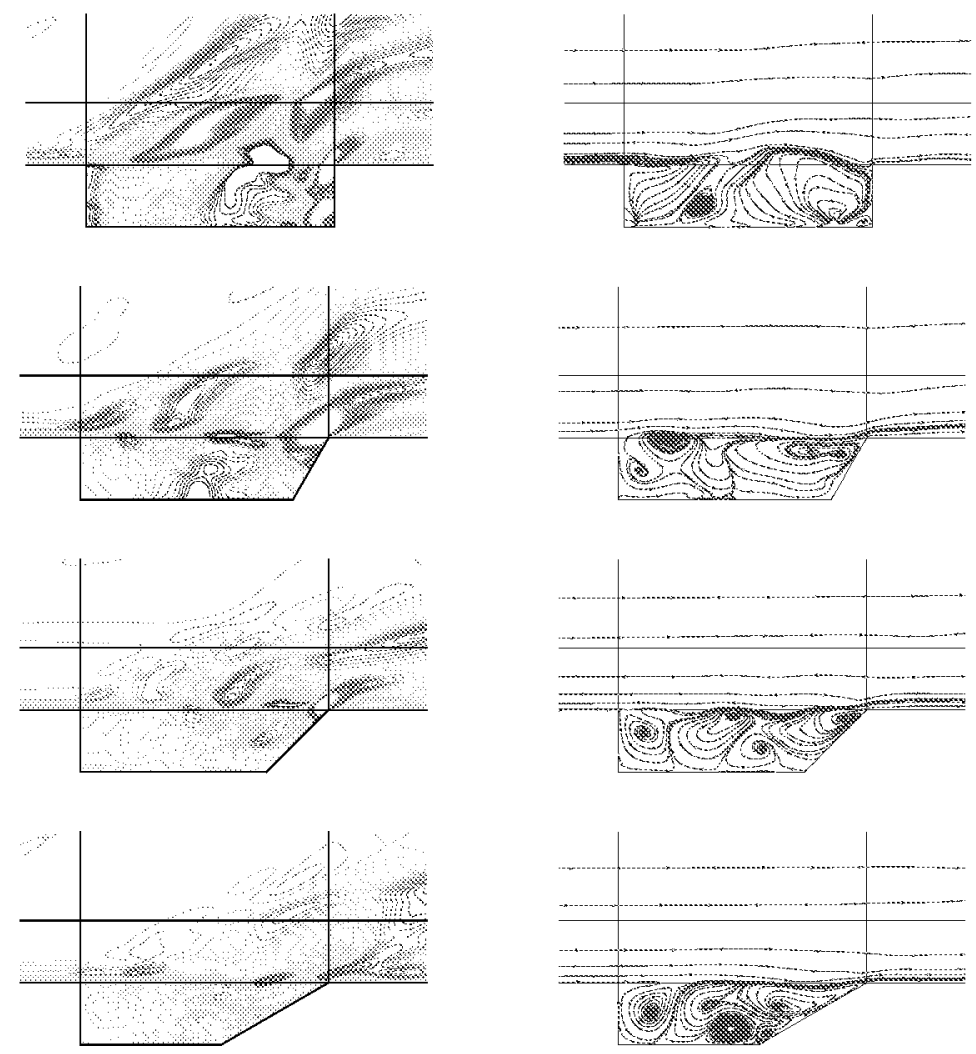

FIG. 5.6. The density contour and the streanline of the non-reactive flows: the left column shows the density contour for $90,60,45$ and 30 degree walls from top to bottom and the right column shows the corresponding streamlines at $t=2.43 \mathrm{~ms}$. The maximum contour level is 1.8 and the minimum 0.5 with the level step size 50 .

aft wall (30 degree in this case) maintains more water than the 90 degree wall at a given time. The figure also shows that the lower angled aft wall holds the flame (water in this case) longer than the 90 degree wall - in the last figure in Figure 5.7 at $t=2.26 \mathrm{~ms}$, the most water is expelled and the only the small amount is left in the left corner while the 30 degree wall cavity holds the water still throughout the cavity. These results imply that the flame-holding efficiency can be increased by lowering the angle of the aft wall of the cavity.

Appendix A. The similarity transform matrices and the eigenvalues of the inviscid flux with chemical species.

\section{Air model without combustion}

First consider the ideal gas composed of two chemically non-reactive species (for the ideal mono-atomic gas $\Lambda$ the diagonal matrix and $S$, the diagonalizer, were given in [15]). $\Lambda$ is given by

$$
\Lambda=\operatorname{diag}(\vec{U} \cdot \vec{n}+c, \vec{U} \cdot \vec{n}, \vec{U} \cdot \vec{n}, \vec{U} \cdot \vec{n}-c, \vec{U} \cdot \vec{n}, \vec{U} \cdot \vec{n}),
$$

where $\vec{U}=(u, v), \vec{n}=\left(n_{x}, n_{y}\right)$ is an unit outward normal vector at the interface and $c$ is a local sound speed.

For simplicity we assume that

$$
\rho \int_{0}^{T} \overline{C_{p}}(s) d s-P \sim \rho C_{v} T
$$



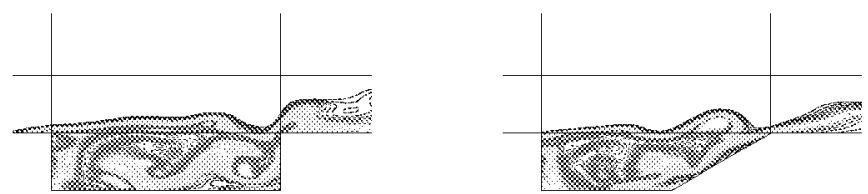

$$
t=0.175 \mathrm{~ms}
$$
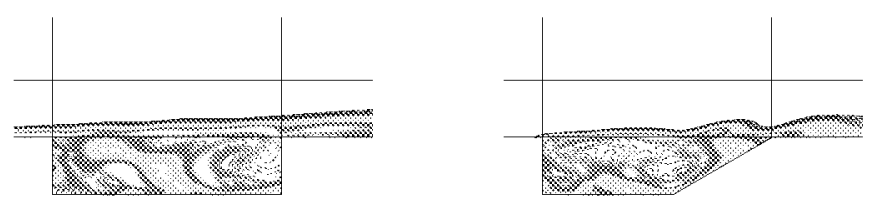

$$
t=0.275 \mathrm{~ms}
$$
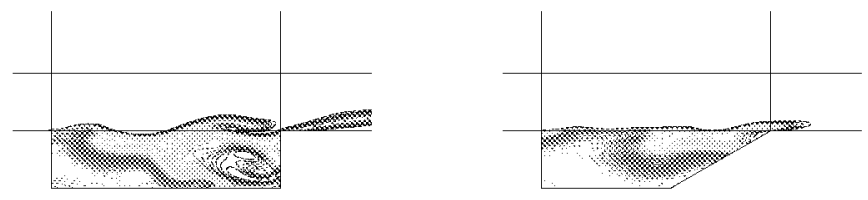

$t=0.945 \mathrm{~ms}$
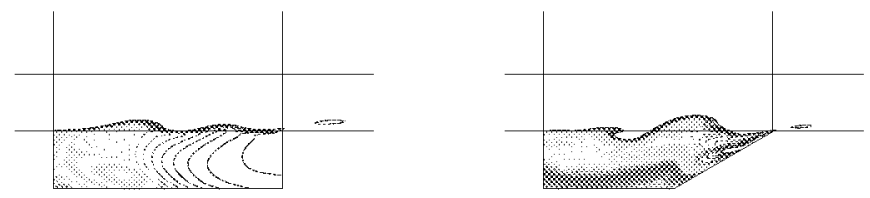

$$
t=2.26 \mathrm{~ms}
$$

FIG. 5.7. The water contour of the reactive flows: the water density contours are given in the lefitigures for 90 degree wall and 30 degree wall in the right figures. From top to bottom the instant times $t$ are $0.175 \mathrm{~ms}, 0.275 \mathrm{~ms}, 0.945 \mathrm{~ms}$ and $2.26 \mathrm{~ms}$. The maximum and minimum contour levels are 0.01 and 0.23 respectively with the number of levels 50.

This form is used only in the analysis, as mentioned in Sec. 3.1, $C_{p_{i}}$ is expressed as a 4 th order polynomial in the temperature $T$. The nonlinear expression of $C_{p_{i}}$ makes it difficult to derive the Jacobian matrices of the fluxes. Our simplifications is a results of assuming small coefficients of the high order terms of the polynomial. In the actual simulations $C_{v}$ is computed appropriately using the empirical law and assumed temperature independent at each linearization step. With this assumption $S$ is given by

$$
S=\left(\begin{array}{cccccc}
1 & 1 & 0 & 1 & 0 & 0 \\
u+c n_{x} & u & -n_{y} & u-c n_{x} & 0 & -n_{y} \\
v+c n_{y} & v & n_{x} & v-c n_{y} & 0 & n_{x} \\
H+c \vec{U} \cdot \vec{n} & \frac{1}{2} \vec{U} \cdot \vec{U} & \vec{U} \cdot \vec{k} & H-c \vec{U} \cdot \vec{n} & \beta c^{2} & \vec{U} \cdot \vec{k}+c \\
f_{1} & a_{12} & 0 & f_{1} & a_{12} & -c \frac{R_{2}}{R_{h}} \\
f_{2} & a_{21} & 0 & f_{2} & a_{21} & c \frac{R_{1}}{R_{h}}
\end{array}\right)
$$

where $H=(E+P) / \rho, R_{i}=R /\left(M_{i} C_{v}\right), R_{h}=R_{1} h_{2}^{0}-R_{2} h_{1}^{0}, R_{v}=\sum_{i=1}^{2} f_{i} R_{i}, \beta=-1 /\left(R_{v}+R_{v}^{2}\right)$, the 

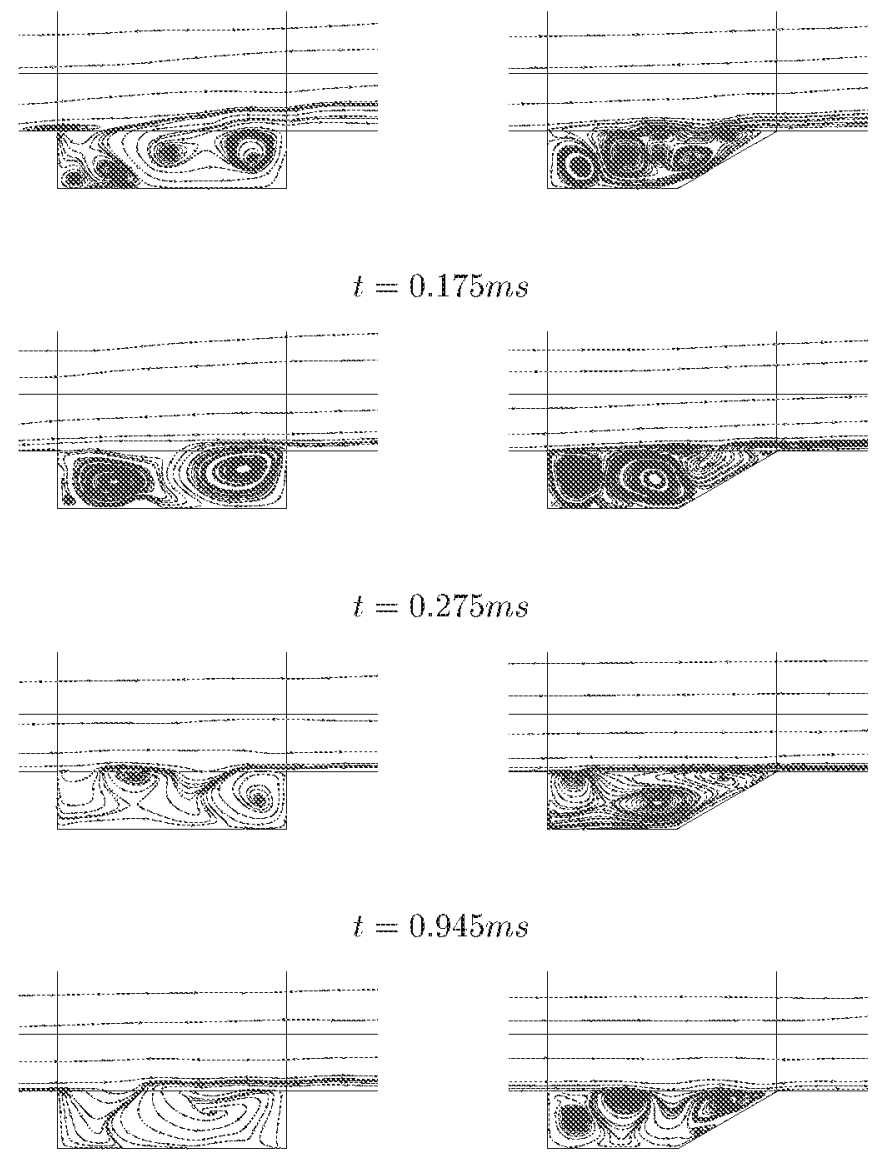

$$
t=2.26 \mathrm{~ms}
$$

FIG. 5.8. The streamlines for the reactive flows: the streamlines for 90 degree wall are shown in the left figures and the 30 degree wall in the right. From top to bottom the times t are $0.175 \mathrm{~ms}, 0.275 \mathrm{~ms}, 0.945 \mathrm{~ms}$ and $2.51 \mathrm{~ms}$.

tangential vector $\vec{k}=\left(-n_{y}, n_{x}\right)$ and

$$
a_{i j}=R_{v} \frac{h_{j}^{0}}{\left(R_{i} h_{j}^{0}-R_{j} h_{i}^{0}\right)}, \quad a_{j i}=R_{v} \frac{h_{i}^{0}}{\left(R_{j} h_{i}^{0}-R_{i} h_{j}^{0}\right)} .
$$

Note that $R_{v}=\gamma-1$ for the mono-atomic ideal gas with $\gamma$, the ratio between the heat capacities $C_{p}$ and $C_{7}$.

\section{Air model with combustion}

Consider now the equations the Euler equations with four reactive species. In this case $\Lambda$ and $S$ are given by

$$
\Lambda=\operatorname{diag}(\vec{U} \cdot \vec{n}+c, \vec{U} \cdot \vec{n}, \vec{U} \cdot \vec{n}, \vec{U} \cdot \vec{n}-\cdots, \vec{U} \cdot \vec{n}, \vec{U} \cdot \vec{n}, \vec{U} \cdot \vec{n}, \vec{U} \cdot \vec{n}),
$$


and

$$
S=\left(\begin{array}{cccccccc}
1 & 1 & 0 & 1 & 0 & 0 & 1 & 1 \\
u+c n_{x} & u & -n_{y} & u-c n_{x} & 0 & -n_{y} & u & u \\
v+c n_{y} & v & n_{x} & v-c n_{y} & 0 & n_{x} & v & v \\
H+c \vec{U} \cdot \vec{n} & \frac{1}{2} \vec{U} \cdot \vec{U} & \vec{U} \cdot \vec{k} & H-c \vec{U} \cdot \vec{n} & \beta c^{2} & \vec{U} \cdot \vec{k}+c & \frac{1}{2} \vec{U} \cdot \vec{U} & \frac{1}{2} \vec{U} \cdot \vec{U} \\
f_{1} & a_{12} & 0 & f_{1} & a_{1234} & R_{1234} & a_{13} & a_{14} \\
f_{2} & a_{21} & 0 & f_{2} & a_{2134} & R_{2134} & 0 & 0 \\
f_{3} & 0 & 0 & f_{3} & a_{3124} & R_{3124} & a_{31} & 0 \\
f_{4} & 0 & 0 & f_{4} & a_{4123} & R_{4123} & 0 & a_{41}
\end{array}\right)
$$

where all the variables are same as in the two species case except that

$$
a_{i j k l}=\epsilon_{i j k l}\left(h_{j}^{0}-h_{k}^{0}+h_{l}^{0}\right) R_{v} / R_{h}
$$

and

$$
R_{i j k l}=-\epsilon_{i j k l}\left(R_{j}-R_{k}+R_{l}\right) c / R_{h}
$$

with

$$
R_{h}=\sum_{i=1}^{4} \epsilon_{i j k l} R_{i}\left(h_{j}^{0}-h_{k}^{0}+h_{l}^{0}\right), \quad i, j, k, l=1,2,3,4, \quad j<k<l,
$$

$\epsilon_{i j k l}$ is the permutation symbol and $R_{v}=\sum_{i=1}^{4} f_{i} R_{i} . \Lambda$ and $S$ are based on the time dependent local spatial quantities at a given time. $f^{ \pm}$is calculated at the interface points at each time.

Appendix B. The symmetrizability of the coefficient matrices of the Navier-Stokes equations with chemical species.

In [1] it had been proven that the coefficient matrices of the Navier-Stokes equations (expressed in the primitive form), of the ideal gas can be simultaneously symmetrized. In $[12,15]$ the same result was demonstrated for the conservative form of the equations. Here we show that it is also true for the NavierStokes equations of the combustible gas with multiple chemical species in two dimension.

Rewrite the linearized Navier-Stokes equations (2.2) in conservative form without the chemical source term as

$$
\frac{\partial q}{\partial t}+\mathbf{A} \frac{\partial q}{\partial x}+\mathbf{B} \frac{\partial q}{\partial y}=\mathbf{C} \frac{\partial^{2} q}{\partial x^{2}}+\mathbf{D} \frac{\partial^{2} q}{\partial x \partial y}+\mathbf{E} \frac{\partial^{2} q}{\partial y^{2}},
$$

where $\mathbf{A}=\frac{\partial F}{\partial q}, \mathbf{B}=\frac{\partial G}{\partial q}, \mathbf{C}=\frac{\partial F_{\nu}}{\partial q_{x}}, \mathbf{D}=\frac{\partial F_{\nu}}{\partial q_{y}}+\frac{\partial G_{\nu}}{\partial q_{w}}$ and $\mathbf{E}=\frac{\partial G_{\nu}}{\partial q_{y}}$. It is sufficient to consider the chemically interacting two chemical species. The coefficient matrices are given by

$$
\mathbf{A}=\left(\begin{array}{cccccc}
0 & 1 & 0 & 0 & 0 & 0 \\
\chi-u^{2} & \left(2-R_{v}\right) u & -R_{v} v & R_{v} & \psi_{1} & \psi_{2} \\
-u v & v & u & 0 & 0 & 0 \\
u(\chi-H) & H-R_{v} u^{2} & -R_{v} u v & \left(1+R_{v}\right) u & u \psi_{1} & u \psi_{2} \\
-u f_{1} & f_{1} & 0 & 0 & u & 0 \\
-u f_{2} & f_{2} & 0 & 0 & 0 & u
\end{array}\right)
$$




$$
\begin{aligned}
& \mathbf{B}=\left(\begin{array}{cccccc}
0 & 0 & 1 & 0 & 0 & 0 \\
-u v & v & u & 0 & 0 & 0 \\
\chi-v^{2} & -R_{v} u & \left(2-R_{v}\right) v & R_{v} & \psi_{1} & \psi_{2} \\
v(\chi-H) & -R_{v} u v & H-R_{v} v^{2} & \left(1+R_{v}\right) v & v \psi_{1} & v \psi_{2} \\
-v f_{1} & 0 & f_{1} & 0 & v & 0 \\
-v f_{2} & 0 & f_{2} & 0 & 0 & v
\end{array}\right) \\
& \mathbf{C}=\left(\begin{array}{cccccc}
0 & 0 & 0 & 0 & 0 & 0 \\
-u \sigma_{1} & \sigma_{1} & 0 & 0 & 0 & 0 \\
-v \sigma_{2} & 0 & \sigma_{2} & 0 & 0 & 0 \\
-\Sigma_{12} & u\left(\sigma_{1}-\sigma_{3}\right) & v\left(\sigma_{2}-\sigma_{3}\right) & \sigma_{3} & \delta_{1} & \delta_{2} \\
0 & 0 & 0 & 0 & 0 & 0 \\
0 & 0 & 0 & 0 & 0 & 0
\end{array}\right) \\
& \mathbf{D}=\left(\sigma_{1}-\sigma_{2}\right)\left(\begin{array}{cccccc}
0 & 0 & 0 & 0 & 0 & 0 \\
-v & 0 & 1 & 0 & 0 & 0 \\
-u & 1 & 0 & 0 & 0 & 0 \\
-2 u v & v & u & 0 & 0 & 0 \\
0 & 0 & 0 & 0 & 0 & 0 \\
0 & 0 & 0 & 0 & 0 & 0
\end{array}\right) \\
& \mathbf{E}=\left(\begin{array}{cccccc}
0 & 0 & 0 & 0 & 0 & 0 \\
-u \sigma_{2} & \sigma_{2} & 0 & 0 & 0 & 0 \\
-v \sigma_{1} & 0 & \sigma_{1} & 0 & 0 & 0 \\
-\Sigma_{21} & u\left(\sigma_{2}-\sigma_{3}\right) & v\left(\sigma_{1}-\sigma_{3}\right) & \sigma_{3} & \delta_{1} & \delta_{2} \\
0 & 0 & 0 & 0 & 0 & 0 \\
0 & 0 & 0 & 0 & 0 & 0
\end{array}\right)
\end{aligned}
$$

where $U_{2}=\frac{1}{2} \vec{U} \cdot \vec{U}, H=\frac{E+P}{\rho}, \sigma_{1}=\frac{2 \mu+\lambda}{\rho}, \sigma_{2}=\frac{\mu}{\rho}, \sigma_{3}=\mu \frac{\widetilde{C}_{p}}{\rho P_{r} C_{v}}, \zeta=\frac{P}{\rho R_{v}}, \chi=R_{v}\left(U_{2}-\zeta\right), \psi_{i}=\zeta R_{i}-$ $R_{v} h_{i}^{0}, \delta_{i}=-h_{i} f_{i} \frac{\mu}{\rho S_{c}}, \tilde{\delta}_{i}=-\frac{\delta_{i}}{\sigma_{3}}, \xi=\frac{E}{\rho}+\sum_{i=1}^{2}\left(h_{i}-\tilde{\delta}_{i}\right) f_{i}, \theta=\xi-2 U_{2}$ and $\Sigma_{j k}=\sigma_{j} u^{2}+\sigma_{k} v^{2}+\sigma_{3} \theta$.

To find the symmetrizer for $\mathbf{A}, \mathbf{B}, \mathbf{C}, \mathbf{D}$ and $\mathbf{E}$, we first consider the similarity transform matrix $\mathbf{S}_{P}$ of C such that

$$
\mathrm{S}_{P}^{-1} \mathrm{CS}_{P}=\Lambda_{C}
$$

where $\Lambda_{C}$ is a diagonal matrix composed of the eigenvalues of $C$. The subscript $P$ denotes that this matrix is adopted from the parabolic portion of the equations [1]. The diagonal matrix $\Lambda_{C}$ of $C$ is given by

$$
\Lambda_{C}=\left(\begin{array}{cccccc}
0 & 0 & 0 & 0 & 0 & 0 \\
0 & \sigma_{1} & 0 & 0 & 0 & 0 \\
0 & 0 & \sigma_{2} & 0 & 0 & 0 \\
0 & 0 & 0 & \sigma_{3} & 0 & 0 \\
0 & 0 & 0 & 0 & 0 & 0 \\
0 & 0 & 0 & 0 & 0 & 0
\end{array}\right) .
$$



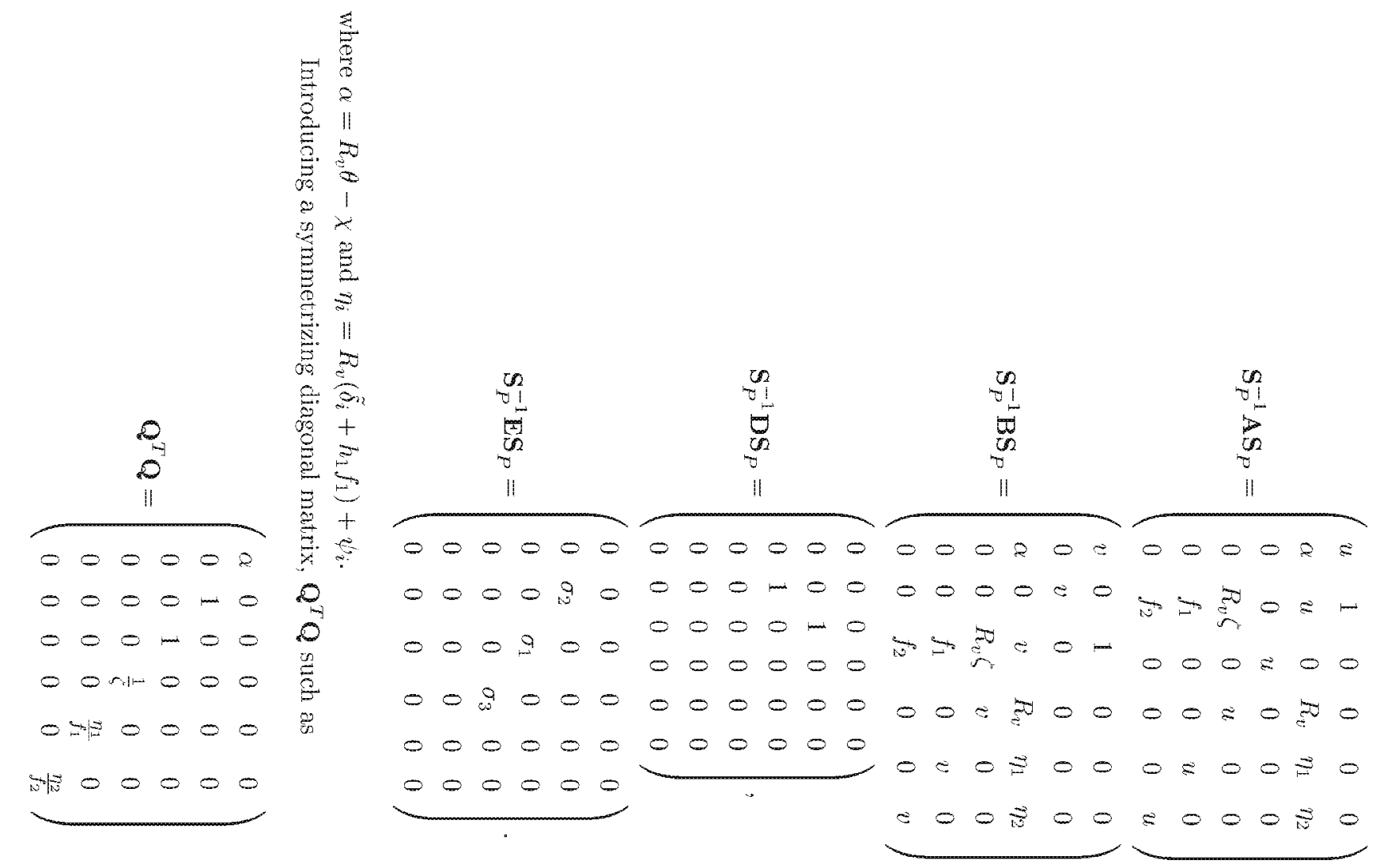
we have symmetrized all the coefficient matrices, i.e.

$$
\begin{aligned}
& \mathrm{Q}^{T} \mathrm{QS}_{P}^{-1} \mathbf{A S}_{P}=\left(\mathbf{Q}^{T} \mathrm{QS}_{P}^{-1} \mathrm{AS}_{P}\right)^{T}, \\
& \mathrm{Q}^{T} \mathrm{QS}_{P}^{-1} \mathbf{B S}_{P}=\left(\mathrm{Q}^{T} \mathrm{QS}_{P}^{-1} \mathrm{BS}_{P}\right)^{T}, \\
& \mathrm{Q}^{T} \mathrm{QS}_{P}^{-1} \mathrm{CS}_{P}=\left(\mathrm{Q}^{T} \mathrm{QS}_{P}^{-1} \mathrm{CS}_{P}\right)^{T}, \\
& \mathrm{Q}^{T} \mathrm{QS}_{P}^{-1} \mathbf{D S}_{P}=\left(\mathrm{Q}^{T} \mathrm{QS}_{P}^{-1} \mathrm{DS}_{P}\right)^{T}, \\
& \mathbf{Q}^{T} \mathrm{QS}_{P}^{-1} \mathbf{E S}_{P}=\mathrm{S}_{P}^{-1} \mathbf{E S}_{P} .
\end{aligned}
$$

\section{Appendix C. Constants for Chemical Models.}

Here we provide constants used in the chemical model for the current numerical experiment. Table I gives the constants used to get the approximation of the specific heat $C_{p i}$ of $i^{\text {th }}$ species in the 4 th order polynomial of $T$, i.e.

$$
C_{p i}=\left(c_{1}+T\left(c_{2}+T\left(c_{3}+T\left(c_{4}+c_{5} T\right)\right)\right)\right) R / M_{i}
$$

where $R$ is a gas constant, and $M_{i}$ is a molecular weight of $i^{\text {th }}$ species [16].

TABLE I

Coefficients for the approximotion of the specific heat $C_{p r}$

\begin{tabular}{l||rrrr}
\hline & $\mathrm{O}_{2}$ & $\mathrm{H}_{2}$ & $\mathrm{H}_{2} \mathrm{O}$ & $\mathrm{N}_{2}$ \\
\hline$c_{1}(1 /$ mole $)$ & 3.0809 & 3.4990 & 3.4990 & 3.1459 \\
$c_{2}(1 /$ mole $)$ & $0.16962 \mathrm{E}-2$ & $-0.18651 \mathrm{E}-3$ & $0.14878 \mathrm{E}-2$ & $0.99154 \mathrm{E}-3$ \\
$c_{3}(1 /$ mole $)$ & $-0.76334 \mathrm{E}-6$ & $0.46064 \mathrm{E}-6$ & $0.87544 \mathrm{E}-7$ & $-0.22912 \mathrm{E}-6$ \\
$c_{4}(1 /$ mole $)$ & $0.17140 \mathrm{E}-9$ & $-0.13157 \mathrm{E}-9$ & $-0.11499 \mathrm{E}-9$ & $0.12181 \mathrm{E}-10$ \\
$c_{5}(1 /$ mole $)$ & $-0.14116 \mathrm{E}-13$ & $0.11679 \mathrm{E}-13$ & $0.13490 \mathrm{E}-13$ & $0.11024 \mathrm{E}-14$ \\
\hline
\end{tabular}

Table II gives the molecular weight and specific enthalpy for each chemical species and Table III gives the reference dynamic viscosity, temperature constants $T$ and $S$ in Wilke's law [21].

TABLE II

Moleculor weights and specific enthalpy

\begin{tabular}{l|rrrr}
\hline & $\mathrm{O}_{2}$ & $\mathrm{H}_{2}$ & $\mathrm{H}_{2} \mathrm{O}$ & $\mathrm{N}_{2}$ \\
\hline \hline$M(1 /$ mole $)$ & 32.000 & 2.016 & 18.016 & 28.016 \\
\hline$h^{0}($ Joule $/ \mathrm{kg})$ & -272918.21 & -4280070.46 & -13973684.55 & -302736.23 \\
\hline
\end{tabular}

TABLE MII

Constants for Walke's law

\begin{tabular}{l|rrrrr}
\hline & $\mathrm{O}_{2}$ & $\mathrm{H}_{2}$ & $\mathrm{H}_{2} \mathrm{O}$ & $\mathrm{N}_{2}$ \\
\hline$\mu_{0}(\mathrm{~kg} / \mathrm{m} / \mathrm{sec})$ & $0.1919 \mathrm{E}-4$ & $0.08411 \mathrm{E}-4$ & $0.1703 \mathrm{E}-4$ & $0.1663 \mathrm{E}-4$ \\
\hline$T_{0}(K)$ & 273.111 & 273.111 & 416.667 & 273.111 \\
\hline$S(K)$ & 138.889 & 96.6667 & 861.111 & 106.667 \\
\hline
\end{tabular}




\section{References}

[1] S. ABARBANEL AND D. GOTTLIEB, Optimal Time Spliting Methods for the Navier Stokes Equations in Two And Three Space Variables, Journal of Computational Physics, 41-1 (1981)

[2] A. BEN-YAKAR AND R. K. HANSON, Cavity flameholders for ignition and flame stabilization in scramjets - Review and experimental study, American Institute of Aeronautics and Astronautics, 98$3122(1998)$

[3] R. A. BAURLE AND M. R. GRUBER, A Study of Recessed Cavity Flowfields for Supersonic Combustion Applications, American Institute of Aeronautics and Astronautics, 98-0938 (1998)

[4] M. H. CARPENTER, J. NORDSTRÖM AND D. GOTTLEB, A Stable And Conservative Interface Treatment of Arbitrary Spatial Accuracy, Journal of Computational Physics, 148-2 (1999), pp. 341-365

[5] M. H. CARPENTER, D. GOTTLIEB AND C. W. SHU, On the conservation and convergence to weak solutions of global schemes, ICASE 2001-44 (2001)

[6] D. L. DAVIS AND R. D. W. BOWERSOX, Stirred Reactor Analysis of Cavity Flame Holders for Scramjets American Institute of Aeronautics and Astronautics 97-3274 (1997)

[7] W. S. DON AND D. GOTTLIEB, Spectral Simulations of Supersonic Reactive Flows, SIAM Journal of Numerical Analysis, 35 (1998), pp. 2370

[8] W. S. DON AND D. GOTTLIEB, High Order Methods for Complicated Flows Interacting with Shock Waves, American Institute of Aeronautics and Astronautics, $97-0538$ (1997)

[9] W. S. DON AND C. QLILLEN, Numerical simulation of Reactive Flow, Part I : Resolution, Journal of Computational Physics, 122 (1995), pp. 244-265

[10] D. FUNARO AND D. GOTTLIEB, A new method of imposing boundary conditions in pseudospectral approximations of hyperbolic equations, Mathematical Computing, 51 (1998), pp. 599-611

[11] D. FLNARO AND D. GOTTLIEB, Convergence results for pseudospectral approximations of hyperbolic systems by a penalty type boundary treatment, Mathematical Computing, 57 (1991), pp. 585-596

[12] A. HARTEN, High resolution schemes for hyperbolic conservation laws, Journal of Computational Physics, 49 (1983), pp. 3à7-393

[13] J. S. HESTHAVEN AND D. GOTTLIEB, Stable Penalty Method for the Compressible Navier-Stokes Equations. I. Open Boundary Conditions, SIAM Journal of Scientific Computing, 17-3 (1996), pp. 579612

[14] J. S. HESTHAVEN, A Stable Penalty Method for the Compressible Navier-Stokes Equations. II. OneDimensional Domain Decomposition Schemes, SIAM Journal of Scientific Computing, 18-3 (1997), pp. 658-685

[15] J. S. HESTHAVEN, A Stable Penalty Method for the Compressible Navier-Stokes Equations. III. Multi Dimensional Domain Decomposition Schemes, SIAM Journal of Scientific Computing, 20-1 (1999), pp. $62-93$

[16] B. J. MCBRIDE, S. HEIMEL, J. G. EHLERS AND S. GORDON, Thermodynamic Properties to $6000^{\circ} \mathrm{K}$ for 210 Substances Involving the First 18 Elements, NASA SP-3001 (1963)

[17] J. NORDSTRÖM AND M. H. CARPENTER, High order finite difference methods, multidimensional linear problems and curvilinear coordinates, Journal of Computational Physics, 148 (1999), pp. 621

[18] J. NORDSTRÖM AND M. H. CARPENTER, High-Order Finite Difference Methods, Multidimensional Linear Problems, and Curvilinear Coordinates, Journal of Computational Physics, 173 (2001), pp. 149

[19] D. P. RIZZETTA, Numerical Simulation of Supersonic Flow Over a Three-Dimensional Cavity, American Institute of Aeronautics and Astronautics, 26-7 (1988), pp. 799 
[20] H. VANDEVEN, Family of spectral filters for discontinuous problems, Journal of Scientific Computing, 24 (1992), pp. $37-49$

[21] C. R. WILKE, A viscosity equation for gas mixtures, Chemical Physies, 18-4 (1950), pp. 517-519

[22] X. ZHANG AND J. A. EDWARDS, The effect of trailing edge gometry on cavity flow oscillation driven by a supersonic shear layer, Aeronautical Journal, 102-1013 (1998), pp. 129-136

[23] X. ZHANG, A. RONA AND J. A. EDWARDS, An observation of pressure waves around a shallow cavity, Aeronautical Journal, 214-4 (1998), pp. 771-778 


\begin{tabular}{|c|c|c|c|}
\hline \multicolumn{3}{|c|}{ REPORT DOCUMENTATION PAGE } & $\begin{array}{l}\text { Form Approved } \\
\text { OMB No. 0704-0188 }\end{array}$ \\
\hline \multicolumn{4}{|c|}{ 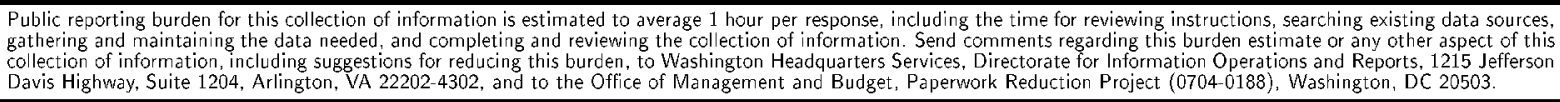 } \\
\hline 1. AGENCY USE ONLY(Leave blank) & $\begin{array}{l}\text { 2. REPORT DATE } \\
\text { August } 2002\end{array}$ & \multicolumn{2}{|c|}{$\begin{array}{l}\text { 3. REPORT TYPE AND DATES COVERED } \\
\text { Contractor Report }\end{array}$} \\
\hline \multicolumn{3}{|c|}{$\begin{array}{l}\text { 4. TITLE AND SUBTITLE } \\
\text { A MULTI-DOMAIN SPECTRAL METHOD FOR SUPERSONIC } \\
\text { REACTIVE FLOWS }\end{array}$} & \multirow[t]{2}{*}{$\begin{array}{l}\text { 5. FUNDING NUMBERS } \\
\text { C NAS1-97046 } \\
\text { WU } 505-90-52-01\end{array}$} \\
\hline \multicolumn{3}{|c|}{$\begin{array}{l}\text { 6. AUTHOR(S) } \\
\text { Wai-Sun Don, David Gottlieb, and Jae-Hun Jung }\end{array}$} & \\
\hline \multicolumn{2}{|c|}{$\begin{array}{l}\text { ICASE } \\
\text { Mail Stop 132C } \\
\text { NASA Langley Research Center } \\
\text { Hampton, VA 23681-2199 }\end{array}$} & & $\begin{array}{l}\text { REPORT NUMBER } \\
\text { ICASE Report No. } 2002-29\end{array}$ \\
\hline \multicolumn{2}{|c|}{$\begin{array}{l}\text { 9. SPONSORING/MONITORING AGENCY NAME(S) AND ADDRESS(E. } \\
\text { National Aeronautics and Space Administration } \\
\text { Langley Research Center } \\
\text { Hampton, VA 23681-2199 }\end{array}$} & $\begin{array}{l}\text { 10. SPONS } \\
\text { AGENC } \\
\text { NASA } \\
\text { ICASI }\end{array}$ & $\begin{array}{l}\text { 10. SPONSORING/MONITORING } \\
\text { AGENCY REPORT NUMBER } \\
\text { NASA/CR-2002-211763 } \\
\text { ICASE Report No. } 2002-29\end{array}$ \\
\hline \multicolumn{4}{|c|}{$\begin{array}{l}\text { 11. SUPPLEMENTARY NOTES } \\
\text { Langley Technical Monitor: Dennis M. Bushnell } \\
\text { Final Report } \\
\text { Submitted to the Journal of Computational Physics. }\end{array}$} \\
\hline \multicolumn{2}{|c|}{$\begin{array}{l}\text { 12a. DISTRIBUTION/AVAILABILITY STATEMENT } \\
\text { Unclassified-Unlimited } \\
\text { Subject Category } 64 \\
\text { Distribution: Nonstandard } \\
\text { Availability: NASA-CASI (301) 621-0390 }\end{array}$} & & 12b. DISTRIBUTION CODE \\
\hline \multicolumn{4}{|c|}{$\begin{array}{l}\text { 13. ABSTRACT (Maximum } 200 \text { words) } \\
\text { This paper has a dual purpose: it presents a multidomain Chebyshev method for the solution of the two-dimensional } \\
\text { reactive compressible Navier-Stokes equations, and it reports the results of the application of this code to the } \\
\text { numerical simulations of high Mach number reactive flows in recessed cavity. The computational method utilizes } \\
\text { newly derived interface boundary conditions as well as an adaptive filtering technique to stabilize the computations. } \\
\text { The results of the simulations are relevant to recessed cavity flameholders. }\end{array}$} \\
\hline \multirow{2}{*}{\multicolumn{3}{|c|}{$\begin{array}{l}\text { 14. SUBJECT TERMS } \\
\text { multi-domain spectral method, penalty interface conditions, supersonic combustor, } \\
\text { recessed cavity flame-holder, compressible Navier-Stokes equations }\end{array}$}} & \begin{tabular}{|c} 
15. NUMBER OF PAGES \\
29
\end{tabular} \\
\hline & & & $\begin{array}{c}\text { 16. PRICE CODE } \\
\text { A03 }\end{array}$ \\
\hline $\begin{array}{l}\text { 17. SECURITY CLASSIFICATION } \\
\text { OF REPORT } \\
\text { Unclassified }\end{array}$ & $\begin{array}{l}\text { 18. SECURITY CLASSIFICATION } \\
\text { OF THIS PAGE } \\
\text { Unclassified }\end{array}$ & $\begin{array}{l}\text { 19. SECURITY CLASSIFICATION } \\
\text { OF ABSTRACT }\end{array}$ & $\begin{array}{l}\text { 20. LIMITATION } \\
\text { OF ABSTRACT }\end{array}$ \\
\hline
\end{tabular}

